\title{
VERA User's Guide for Ex-Core Applications
}

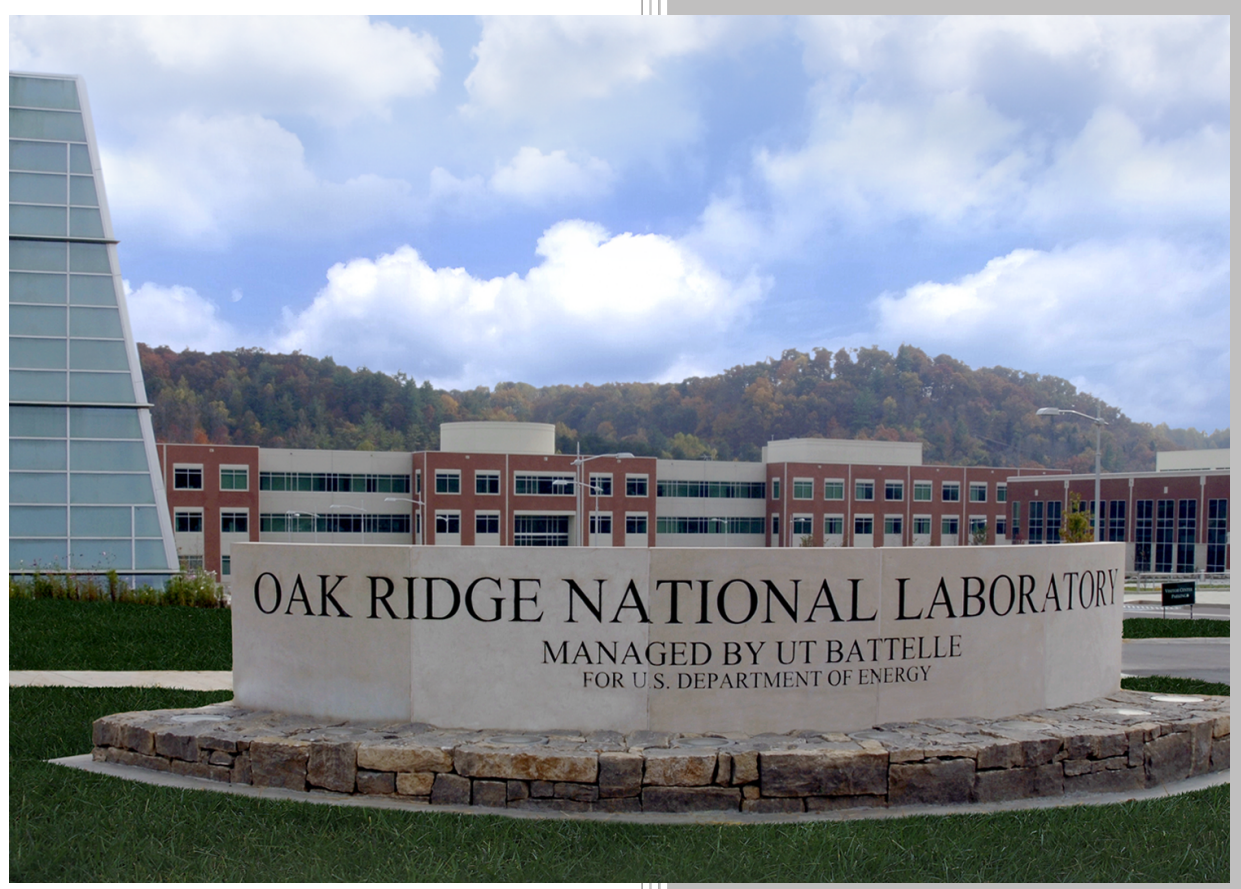

\section{Approved for public release.} Distribution is unlimited.

Eva Davidson

January 2022 


\section{DOCUMENT AVAILABILITY}

Reports produced after January 1, 1996, are generally available free via US Department of Energy (DOE) SciTech Connect.

Website: http://www.osti.gov/scitech/

Reports produced before January 1, 1996, may be purchased by members of the public from the following source:

National Technical Information Service

5285 Port Royal Road

Springfield, VA 22161

Telephone: 703-605-6000 (1-800-553-6847)

TDD: 703-487-4639

Fax: 703-605-6900

E-mail: info@ntis.gov

Website: http://classic.ntis.gov/

Reports are available to DOE employees, DOE contractors, Energy Technology Data Exchange representatives, and International Nuclear Information System representatives from the following source:

Office of Scientific and Technical Information

PO Box 62

Oak Ridge, TN 37831

Telephone: 865-576-8401

Fax: 865-576-5728

E-mail: report@osti.gov

Website: http://www.osti.gov/contact.html

This report was prepared as an account of work sponsored by an agency of the United States Government. Neither the United States Government nor any agency thereof, nor any of their employees, makes any warranty, express or implied, or assumes any legal liability or responsibility for the accuracy, completeness, or usefulness of any information, apparatus, product, or process disclosed, or represents that its use would not infringe privately owned rights. Reference herein to any specific commercial product, process, or service by trade name, trademark, manufacturer, or otherwise, does not necessarily constitute or imply its endorsement, recommendation, or favoring by the United States Government or any agency thereof. The views and opinions of authors expressed herein do not necessarily state or reflect those of the United States Government or any agency thereof. 
Reactor and Nuclear Systems Division

\title{
VERA User's Guide for Ex-core Applications
}

\author{
Eva Davidson
}

Date Published: January 2022

Prepared by

OAK RIDGE NATIONAL LABORATORY

Oak Ridge, TN 37831-6283

managed by

UT-Battelle, LLC

for the

US DEPARTMENT OF ENERGY

under contract DE-AC05-00OR22725 



\section{CONTENTS}

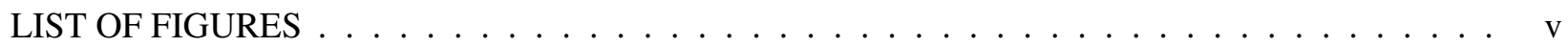

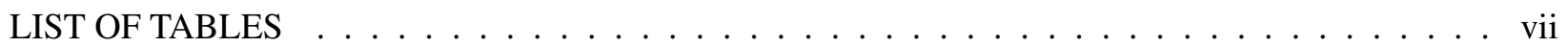

LIST OF INPUTS, OUTPUTS AND SCRIPTS $\ldots \ldots \ldots \ldots \ldots \ldots \ldots$. . . . . . $\ldots \ldots$

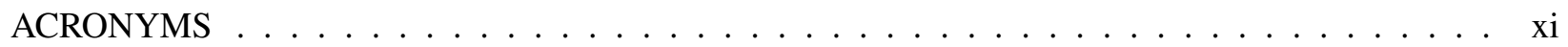

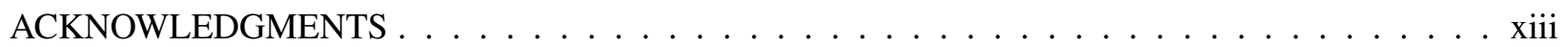

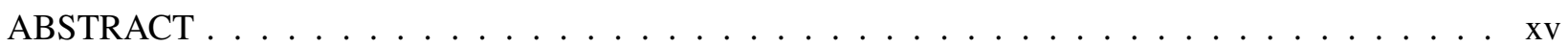

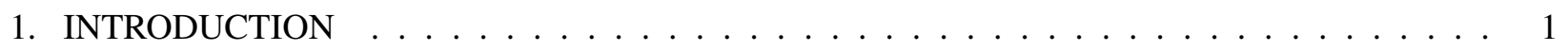

2. RUNNING VERA USING VERARUN $\ldots \ldots \ldots \ldots \ldots \ldots \ldots \ldots$

2.1 SETTING UP MPACT AND SHIFT PROCESSORS $\ldots \ldots \ldots \ldots \ldots$

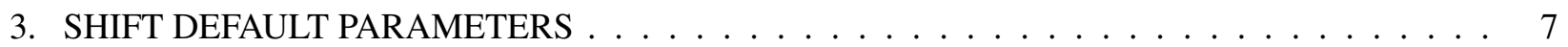

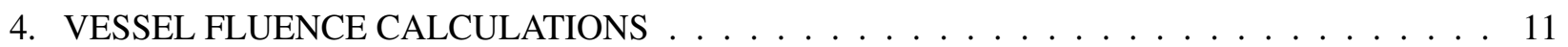

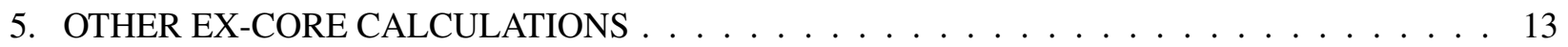

5.1 SETTING UP THE OMNIBUS EX-CORE GEOMETRY $\ldots \ldots \ldots \ldots$

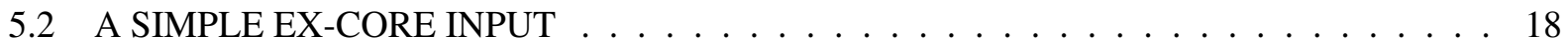

5.3 SMR EX-CORE INPUT WITH A DETECTOR . . . . . . . . . . . . . . . . . . 21

5.4 AUTOMATED GENERATION OF THE BIOSHIELD AND EX-CORE DETECTORS . . . 28

5.5 RUNNING VERA WITH AN EX-CORE FILE . . . . . . . . . . . . . . . . . 32

6. PRE- AND POST-PROCESSING INPUTS AND OUTPUTS . . . . . . . . . . . . . . . . . 35

6.1 CHECKING FOR GEOMETRY ERRORS DURING EX-CORE INPUT SETUP . . . . . . 35

6.2 GENERATING PLOTS AFTER RUNNING VERA . . . . . . . . . . . . . . . . . . . . . 39

6.2 .1 Using VERAView To Examine Pin Adjoints . . . . . . . . . . . . . . . . . . . 39

6.2 .2 Custom Python Scripts . . . . . . . . . . . . . . . . . . . . . 40

6.3 POST-PROCESSING CELL TALLIES AFTER RUNNING VERA . . . . . . . . . . . . . . 42

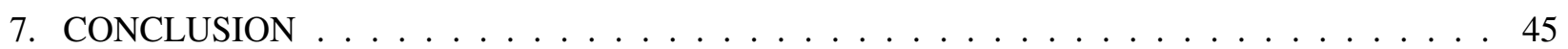

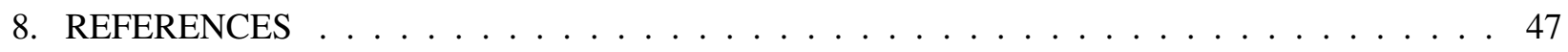





\section{LIST OF FIGURES}

1 VERAView screen with vessel fluence output. . . . . . . . . . . . . . . . . 12

2 Example of (a) ex-core detector within a detector well in the bioshield and (b) the spatial fission source. . . . . . . . . . . . . . . . . . . . . 13

3 Example of a geometry set up with a single surveillance capsule and a power range detector. 14

4 Full core model. . . . . . . . . . . . . . . . . . . . . . . . . . . . . . . 14

5 Quarter core quadrants modeled with MPACT and Shift for quarter core problems. . . . . 15

6 Simple ex-core region for an SMR. . . . . . . . . . . . . . . . . . 18

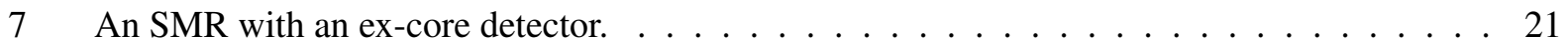

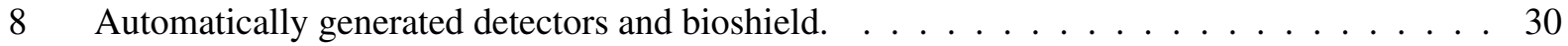

9 An example of a plot output from plot.py. . . . . . . . . . . . . . . . . . 39

10 Pin adjoint plots generated with VERAView. . . . . . . . . . . . . . . . . 40

11 Integrated in-core and ex-core geometry with the adjoint flux overlaid on the geometry. . . . 42 



\section{LIST OF TABLES}

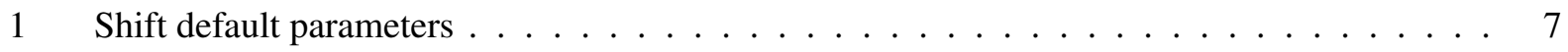

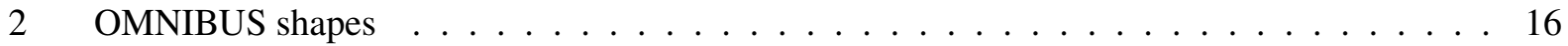





\section{LIST OF INPUTS, OUTPUTS AND SCRIPTS}

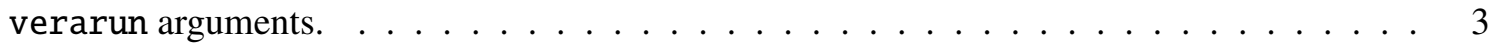

Simple vessel fluence VERA input. . . . . . . . . . . . . . . . . . . . 11

VERA vessel fluence input with additional parameters. . . . . . . . . . . . . . 11

Blocks of input in Omnibus ex-core file . . . . . . . . . . . . . . . . . . . . 17

Simple ex-core file. . . . . . . . . . . . . . . . . . . . . . 19

Ex-core file with bioshield and detector. . . . . . . . . . . . . . . . . 22

VERA input file for automated detector generation. . . . . . . . . . . . . . 28

Default materials in automatically generated ex-core file. . . . . . . . . . . . . . . . 29

[TALLY] block automatically generated in the ex-core file. . . . . . . . . . . . . . 31

10 [SHIFT] block in VERA input for running ex-core calculations with an ex-core file. . . . . 32

11 Revised ex-core file for geometry visualization and error-checking. . . . . . . . . . . 35

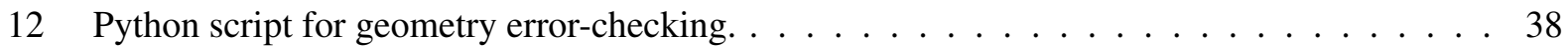

13 Python script for viewing the geometry and adjoint after running VERA . . . . . . . . . 41

14 Python script for viewing the geometry and adjoint after running VERA. . . . . . . . . . . 42 



\section{ACRONYMS}

CADIS Consistent Adjoint Driven Importance Sampling

EFPD effective full power day

HDF5 hierarchical data formats version 5

GG General Geometry

ORNL Oak Ridge National Laboratory

SMR small modular reactor

VERA Virtual Environment for Reactor Applications

VR variance reduction 



\section{ACKNOWLEDGEMENTS}

The author would like to thank Andrew Godfrey for his guidance and for providing feedback on the scripts required for streamlining VERA ex-core calculations.

The author would also like to acknowledge the entire Exnihilo and MPACT development teams for their patience and user support while the author ran numerous ex-core calculations. The author also appreciates the assistance received from Tara Pandya and Katherine Royston of the Exnihilo development team in setting up the appropriate scripts to plot and display various pieces of information from the output. 



\begin{abstract}
The Virtual Environment for Reactor Applications, or VERA, allows users to set up models to calculate time-dependent and fully coupled solutions for ex-core quantities of interest such as vessel and coupon fluence, and detector responses for multiple statepoints and cycles. MPACT and COBRA-TF together perform in-core transport calculations with temperature feedback while Shift performs the fluence and detector response calculations in the ex-core region. The in-core region is modeled using VERA's native input format and the ex-core region is defined using Shift's general geometry package, also known as Omnibus General Geometry. Fixed source ex-core calculations with Shift can be run in forward mode without advanced variance reduction (VR) methods, or with Consistent Adjoint Driven Importance Sampling (CADIS), which is an automated VR method.
\end{abstract}

This document serves as a guide for setting up inputs, running ex-core calculations and post-processing the results. 



\section{INTRODUCTION}

Advanced simulation methods in VERA (Turner et al. [2016]) allow users to set up models to calculate time-dependent and fully coupled solutions for coupon fluence, pressure vessel fluence, and ex-core detector responses at multiple statepoints and for multiple cycles. The in-core transport with temperature feedback is performed by coupled MPACT (Collins et al. [2016]) and COBRA-TF (Avramova [2009]) calculations, and the fluence and detector response calculations in the ex-core region are performed by Shift (Pandya et al. [2016]). The ex-core region is defined using Shift's general geometry package, also known as Omnibus General Geometry (GG) (Johnson et al. [2020]), while the in-core geometry is defined using the standard VERA input (Palmtag and Godfrey [2015]). Fixed source ex-core calculations in Shift can be run in forward mode without advanced variance reduction (VR) methods, or with Consistent Adjoint Driven Importance Sampling (CADIS) (Wagner and Haghighat [1998]), which is an automated VR method. Space- and energy-dependent weight windows for Shift are generated using Denovo, a discrete ordinates solver in the Oak Ridge National Laboratory (ORNL) Exnihilo code suite (Johnson et al. [2020]).

The VERA input set up to perform in-core calculations with MPACT can be used to run ex-core calculations with Shift. Once the VERA input has been set up, no additional user effort is required to model the core or fuel in great detail in order to perform ex-core calculations. Subsequently, vessel fluence calculations can be performed with very little user input, but more complicated ex-core geometry must be modeled using the GG input format.

This document serves as a user's guide for setting up and running ex-core calculations with VERA as well as post-processing the results. 



\section{RUNNING VERA USING VERARUN}

VERA ex-core calculations can be invoked with verarun. The user can gain more information regarding all the input arguments for verarun by typing the following into the terminal window:

verarun

This command will show the user a list of the arguments (Listing 1) and a description of what each argument would accomplish.

\section{Listing 1. verarun arguments.}

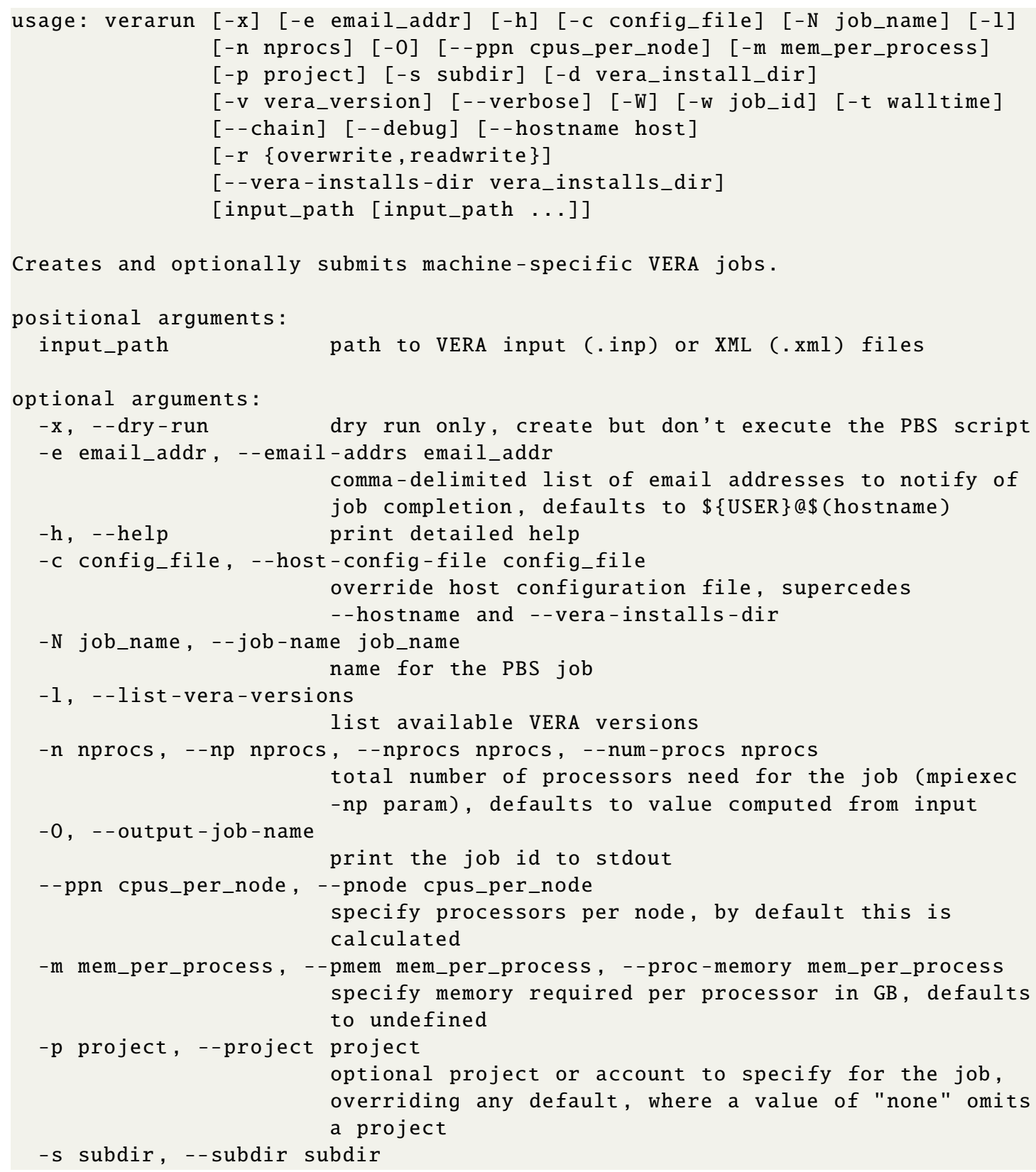




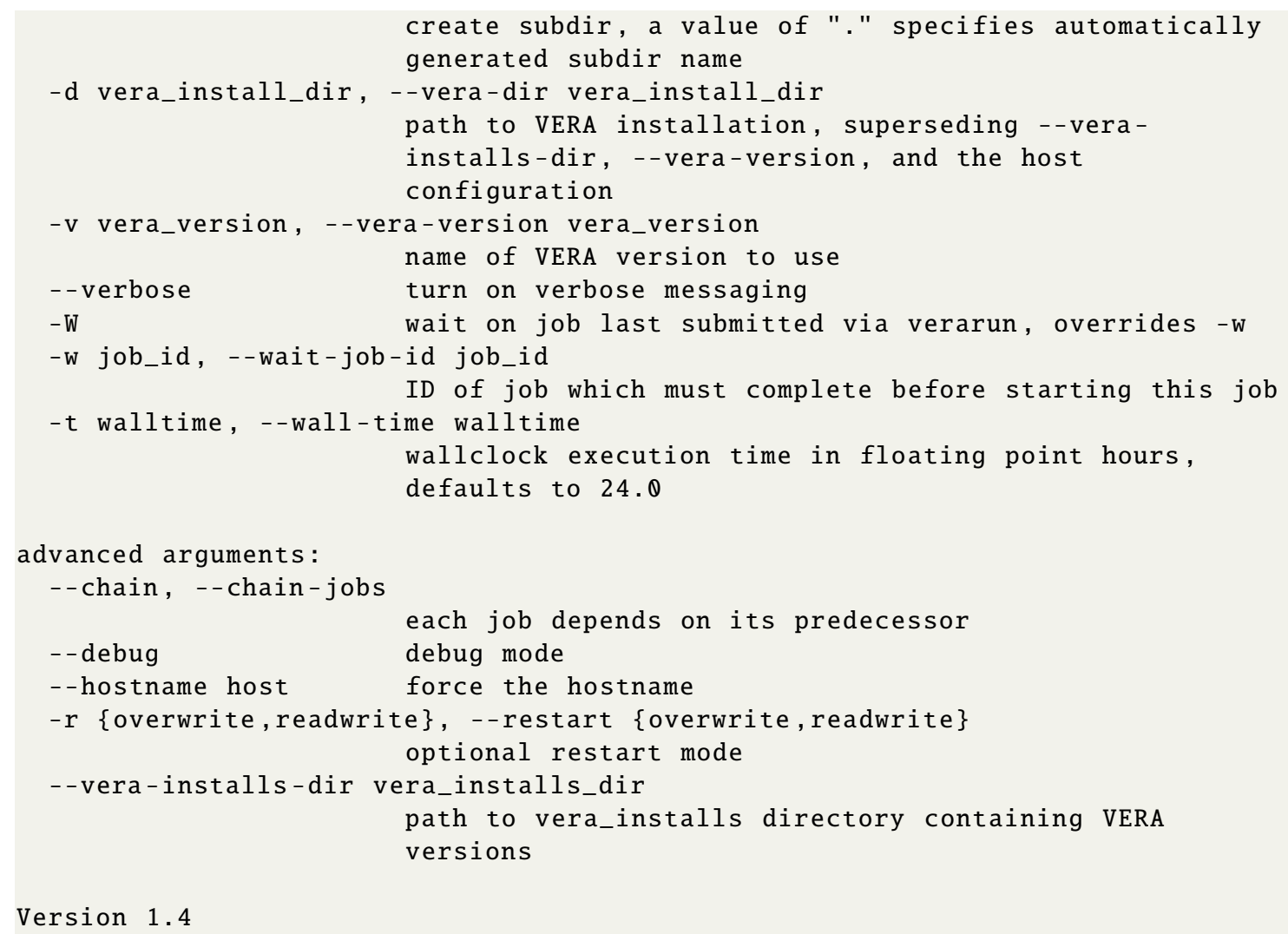

Version 1.4

When running ex-core problems with a supplemental Omnibus ex-core file, ensure that both the VERA input and ex-core files are in the same folder. To run Shift calculations in cadis mode, type in:

verarun vera_input_filename.inp

Note that if there is an Omnibus ex-core file, do not forget to insert excore-filename.excore.xml within the [SHIFT] block in the VERA input using the excore_filename parameter [Pandya et al. [2020]].

\subsection{SETTING UP MPACT AND SHIFT PROCESSORS}

When running ex-core calculations with VERA, the problem should be set up so that MPACT and Shift run on different nodes. Otherwise, it is possible to run out of memory on a node if both MPACT and Shift are sharing that node. For example, if a node has 32 processors, it is good practice to ensure that MPACT is running on a multiple of 32 processors.

The num_space parameter in the [MPACT] block refers to the number of processors MPACT requires for the problem, and it can be toggled to ensure that MPACT runs on its own independent nodes. The num_blocks_i and num_blocks_j parameters in the [SHIFT] block provide information on the number of Shift processors required. Note that these Shift parameters are associated with CADIS, and CADIS is the default method when invoking Shift for ex-core calculations. When using verarun to run a VERA ex-core calculation, the default number of Shift processors is 10 num_blocks_i multiplied by 10 num_blocks_j, which is equal to 100 processors for Shift. However, the user is free to run with any combination of num_blocks_i and num_blocks_j to utilize all the processors available. It must be noted 
that for improved performance and efficiency, it is preferable to keep the num_blocks_i equal to num_blocks_j. The user must also bear in mind that if a cluster with 32 processors per node is being used, and the default 100 processors is used for the Shift calculation when using verarun, then there will be 28 processors that will not be used in the calculation.

Shift ex-core calculations can also be performed without advanced variance reduction. In this case, the user must specify that the problem has to be run in forward mode in the [SHIFT] block. The user can state the total number of processors required for the VERA simulation by typing the following command into the terminal window:

verarun $-\mathrm{n}$ nprocs

The nprocs value is equal to the total number of MPACT and Shift processors. verarun reads the number of MPACT processors required for the problem from the num_space input parameter within the [MPACT] block; the remainder of the processors will be assigned to Shift. 



\section{SHIFT DEFAULT PARAMETERS}

Most hybrid parameters that allow ex-core problems to run efficiently have been set as defaults in the SHIFT . ini file in the build directory, \$HOME/bin/Init. The parameters in the SHIFT.ini file have been set to the optimum values for most problems except for a few parameters that must be specified by the user for their specific problems. Expert users of hybrid methods can toggle some of the default parameters for their specific models. More information regarding these parameters and other parameters for the [SHIFT] block in the VERA input can be found in the manual by Pandya et al. [2020].

Table 1 lists the default parameters within the SHIFT . ini file (Pandya et al. [2020]).

Table 1. Shift default parameters

\begin{tabular}{|c|c|c|c|}
\hline Parameter & Default Value & Value Options & Description \\
\hline adjoint & true & - & $\begin{array}{l}\text { Set to true if running in cadis } \\
\text { mode }\end{array}$ \\
\hline create_unique_pins & false & - & $\begin{array}{l}\text { Currently set to false for all } \\
\text { problems due to memory con- } \\
\text { straints. If set to true, each pin- } \\
\text { cell would have unique compo- } \\
\text { sitions }\end{array}$ \\
\hline dimension & 3 & - & $\begin{array}{l}\text { Spatial dimension of the prob- } \\
\text { lem }\end{array}$ \\
\hline do_transport & true & true/false & $\begin{array}{l}\text { Perform Monte Carlo transport } \\
\text { if set to true; otherwise, exit the } \\
\text { calculation after performing a } \\
\text { raytrace of the geometry }\end{array}$ \\
\hline eq_set & $\mathrm{sc}$ & - & $\begin{array}{l}\text { Solution method or spatial dis- } \\
\text { cretization for hybrid calcula- } \\
\text { tion ( } \mathrm{sc}=\text { step characteristics) } \\
\text { for ex-core calculations }\end{array}$ \\
\hline extend_axial_mesh_size & 5.0 & positive value & $\begin{array}{l}\text { Adjoint axial mesh size in ex- } \\
\text { core region }\end{array}$ \\
\hline global_log & info & $\begin{array}{l}\text { debug/diagnostics/ status/in- } \\
\text { fo/warning/ error/critical }\end{array}$ & Level of global log information \\
\hline max_delta_z & 5.0 & $\begin{array}{l}\text { User can decrease mesh size for } \\
\text { hybrid calculation }\end{array}$ & $\begin{array}{l}\text { Maximum mesh size in } \mathrm{z} \text { di- } \\
\text { rection used for hybrid calcula- } \\
\text { tions }\end{array}$ \\
\hline mesh & 1 & - & $\begin{array}{l}\text { Number of mesh cells per pin- } \\
\text { cell }\end{array}$ \\
\hline mode & $\mathrm{n}$ & $\mathrm{n} / \mathrm{np}$ & $\begin{array}{l}\text { Type of particle to transport } \\
\text { (neutrons/ neutrons and pho- } \\
\text { tons) }\end{array}$ \\
\hline
\end{tabular}




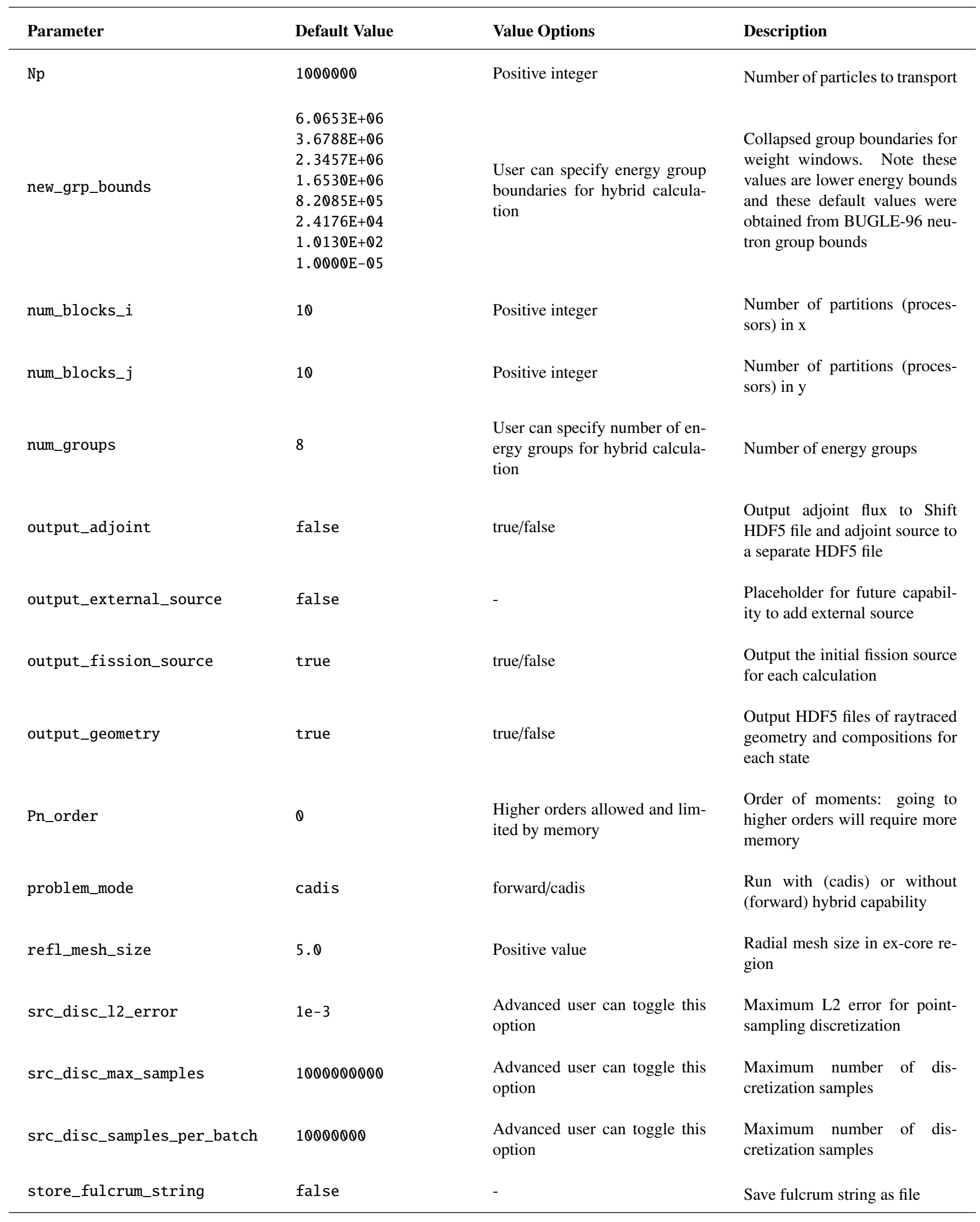




\begin{tabular}{|c|c|c|c|}
\hline Parameter & Default Value & Value Options & Description \\
\hline thermal_energy_cutoff & 10.0 & - & $\begin{array}{l}\text { Cutoff for treatment of } \\
\text { thermal neutrons }\end{array}$ \\
\hline transfer & fiss_src & - & $\begin{array}{l}\text { Information transferred from } \\
\text { MPACT to Shift (currently the } \\
\text { option for ex-core calculations) }\end{array}$ \\
\hline transport & ce & - & $\begin{array}{l}\text { Continuous energy (only option } \\
\text { for ex-core calculations) }\end{array}$ \\
\hline upscatter_subspace_size & 20 & User can specify a higher value & Upscatter solver subspace size \\
\hline upscatter_verbosity & high & - & $\begin{array}{l}\text { Log verbosity for up- } \\
\text { scatter solver }\end{array}$ \\
\hline use_external_source & false & - & $\begin{array}{l}\text { Placeholder for future capabil- } \\
\text { ity that indicates an external } \\
\text { source is being used }\end{array}$ \\
\hline use_fission_source & true & - & $\begin{array}{l}\text { Flag that indicates a fission } \\
\text { source is being used }\end{array}$ \\
\hline use_mpact_spectrum & false & true/false & $\begin{array}{l}\text { Flag for whether to use the } \\
\text { MPACT fission source energy } \\
\text { spectrum to sample for neu- } \\
\text { tron source energies: if false, } \\
\text { nuclide-watt spectrum is used; } \\
\text { the neutron source energies are } \\
\text { assumed to have }{ }^{235} \mathrm{U},{ }^{238} \mathrm{U} \\
{ }^{239} \mathrm{Pu},{ }^{241} \mathrm{Pu} \text { Watt spectra based } \\
\text { on the fission source strength of } \\
\text { these isotopes }\end{array}$ \\
\hline verbosity & low & none/low/medium/high & $\begin{array}{l}\text { Specify how often to print out } \\
\text { particles being transported: low } \\
\text { means less output, and high } \\
\text { means more output }\end{array}$ \\
\hline xs_library & v7-56 & - & $\begin{array}{l}\text { Name of SCALE multigroup } \\
\text { data library file }\end{array}$ \\
\hline
\end{tabular}





\section{VESSEL FLUENCE CALCULATIONS}

Running vessel fluence simulations with VERA is the simplest application for performing ex-core calculations based on the minimal effort required by the user to set up an input. Consider a VERA input that was previously set up or run with other physics options in VERA. A user can perform a vessel fluence calculation on the same input if an additional line is added to the first state block in the VERA input.

\section{[STATE]}

excore_transport on

Next, a user must provide more information regarding the number of meshes and the number of particles they want to run for the vessel fluence calculation using the [SHIFT] block in the VERA input. This input block can be added at the end of the VERA input file. Note that these vessel fluence calculations are run without the bioshield.

As an example, let us review the following input:

Listing 2. Simple vessel fluence VERA input.

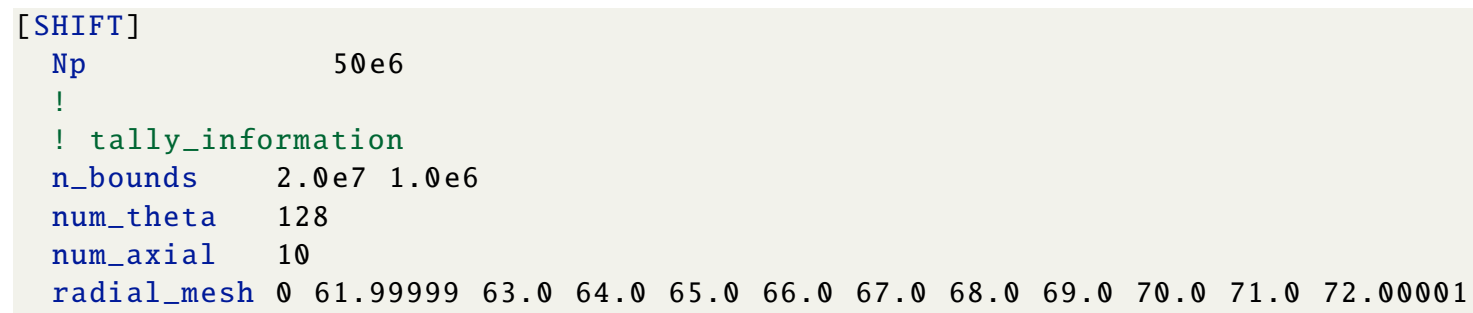

This input shows that this particular case will be run with 50 million particles, and the energy bounds on the tally will range from $2.0 \times 10^{7} \mathrm{eV}$ to $1.0 \times 10^{6} \mathrm{eV}$. The energy bounds must be supplied in descending order. There are 128 meshes along theta and 10 axial meshes. The bounds of the radial mesh are provided from $0 \mathrm{~cm}$ to the outer extent of the vessel. The radial mesh must always start with $0 \mathrm{~cm}$, which is the origin. The second value is then the inner radius of the vessel liner or vessel, whichever the user prefers.

The user can add additional inputs that were provided in the previous Shift default parameters section to their [SHIFT] block. In the following input, the output_adjoint parameter is set to true so that the user can use the python post-processors available with VERA to visualize the adjoint. The default number of processors that a Shift calculation runs on is 100 processors. This can be changed to 400 processors or any other number, as the user prefers. In the input below, the Shift calculation will be performed on 400 processors $(20 \times 20)$.

\section{Listing 3. VERA vessel fluence input with additional parameters.}

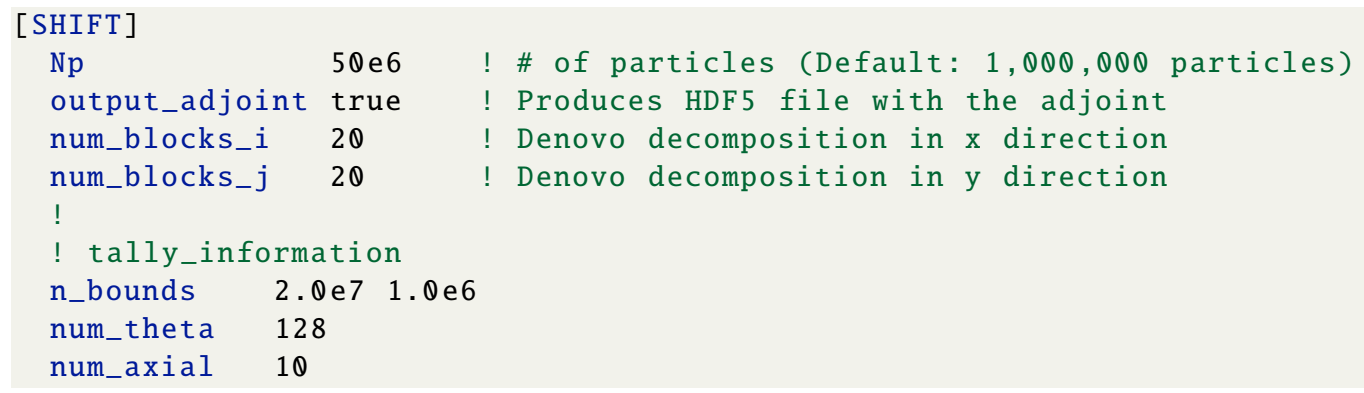


After the Shift parameters are added, the input is ready to be run using verarun. Once the run is complete, the output can be viewed in VERAView by clicking on Load Single New File and selecting the HDF5 output file that ends in your input_filename.h5. Note that there are several HDF5 output files in the run folder; however, the output read by VERAView is the input_filename.h5.

Once the file is loaded, the following screen will be displayed.

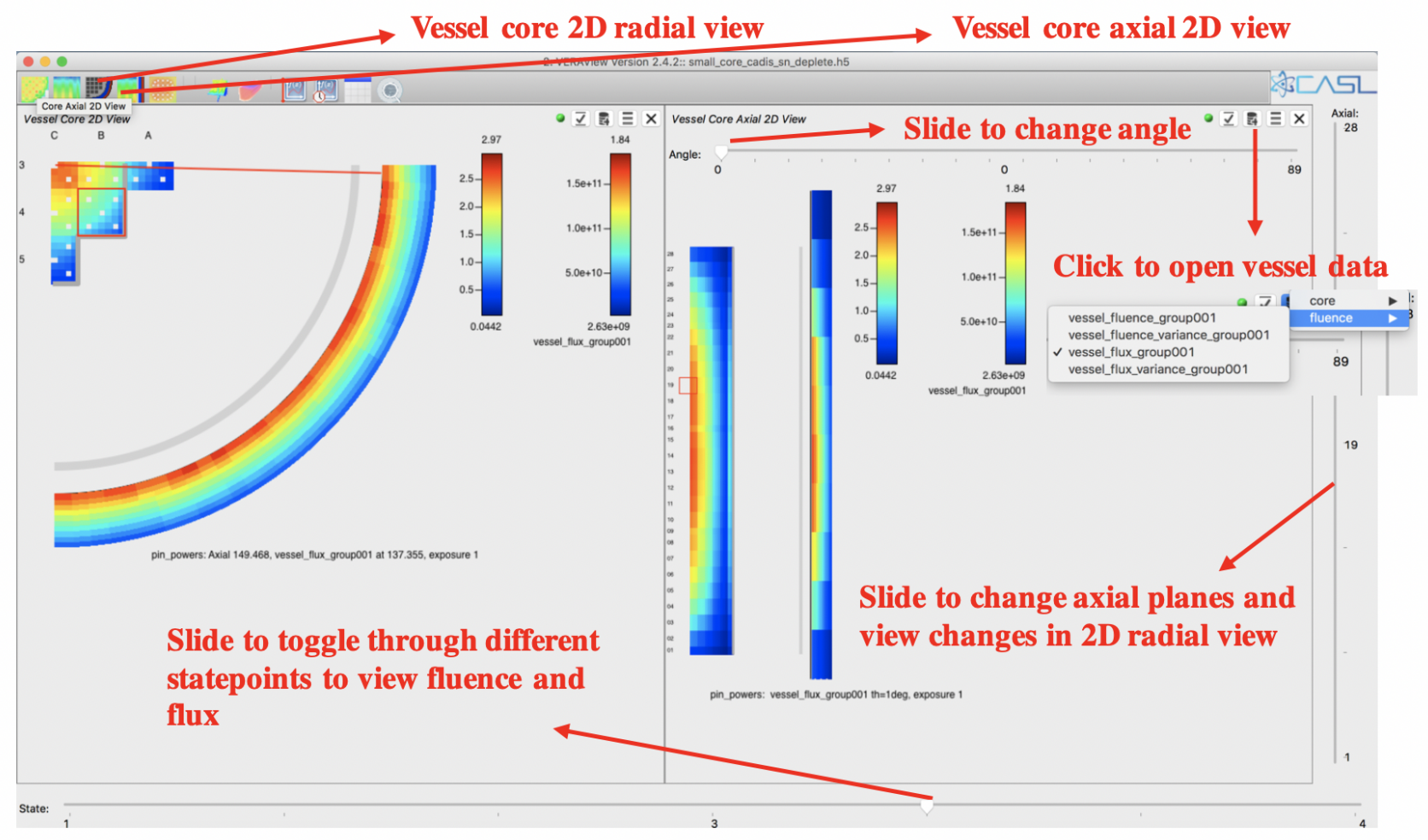

Figure 1. VERAView screen with vessel fluence output.

In this figure, the plot on the left shows the radial view of the pin powers and the vessel flux, while the plot on the right shows an axial view of the pin powers and vessel flux with two different color maps shown for pin powers and the vessel flux. Sliding the bar at the bottom of the screen allows the user to toggle between different statepoints, and sliding the bar at the top of the screen can be used to change the angle. The bar on the right-hand side can be used to toggle between different axial planes. The user can view the vessel fluence or flux by clicking on the "hamburger" icon on the top right corner of the screen and selecting the data to plot. Note that in the figure generated by VERAView, the vessel is not presented at scale and looks like it is really far away from the reactor. VERAView also does not plot the core pads. 


\section{OTHER EX-CORE CALCULATIONS}

To perform ex-core calculations such as detector response beyond the pressure vessel or coupon fluence calculations, the user must set up a separate input to define the ex-core geometry using Omnibus input format. This process enables the user to set up a detailed core model using VERA and to integrate this core model with the Omnibus geometry to define the ex-core problem. Setting up an Omnibus input can be as simple or as complex as the geometry for the region the user is interested in studying. The Omnibus geometry package is called General Geometry (GG), and it is based on the concept of geometric primitives combined with unions and intersections. If the user is familiar with SCALE's KENO or Monte Carlo $\mathrm{N}$-Particle (MCNP) code, then GG-based input should not present a steep learning curve. Figure 2 below shows an ex-core detector response problem set up for Shearon Harris [Smith et al. [2018] and Davidson et al. [2020]]. Figure 3 shows coupons in single surveillance capsules modeled for Watts Bar Unit 1 [Davidson et al. [2018]. Figure 4 shows a full core Watts Bar model [Davidson et al. [2018] with all the ex-core detectors (power range and source range) and surveillance capsules (single and double) off the pads.

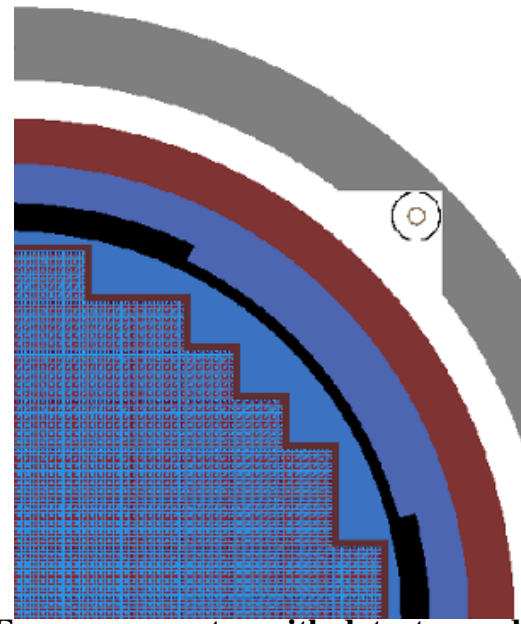

(a) Ex-core geometry with detector and detector well

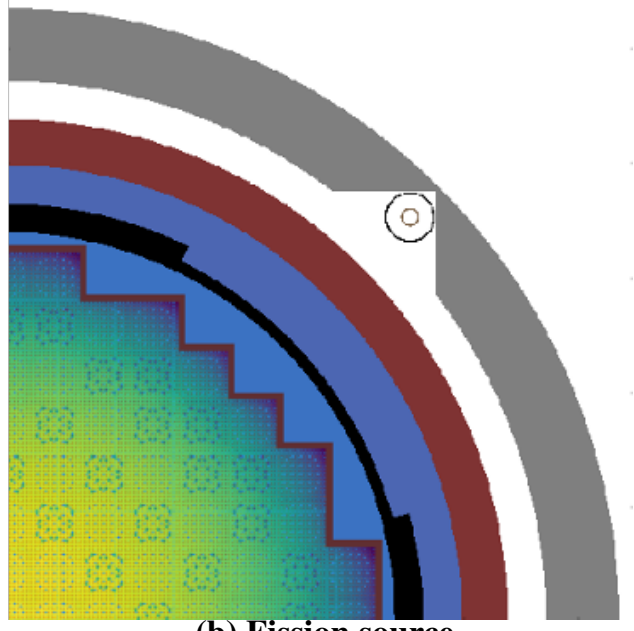

(b) Fission source

Figure 2. Example of (a) ex-core detector within a detector well in the bioshield and (b) the spatial fission source. 


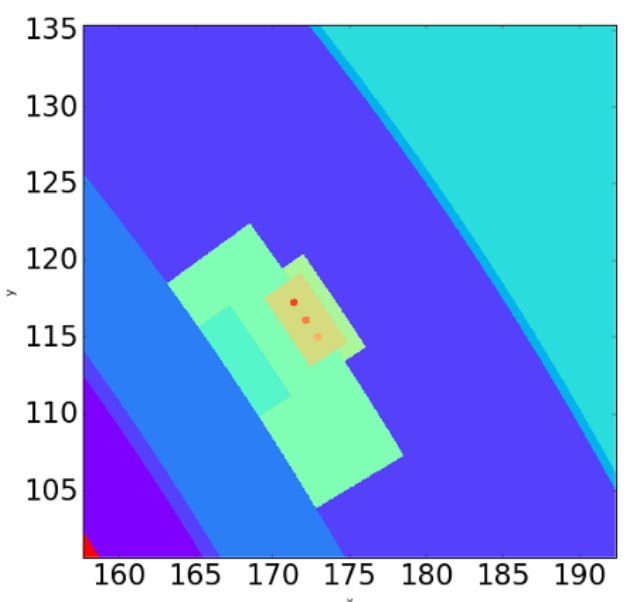

(a) Coupons

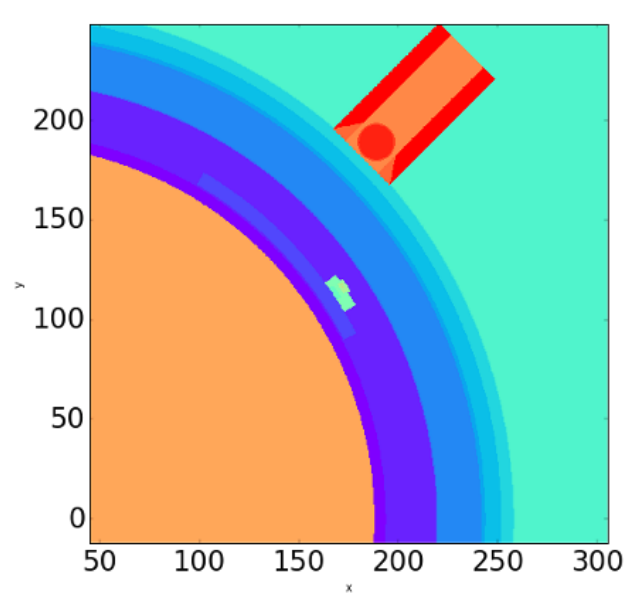

(b) Single surveillance capsule and detector

Figure 3. Example of a geometry set up with a single surveillance capsule and a power range detector.

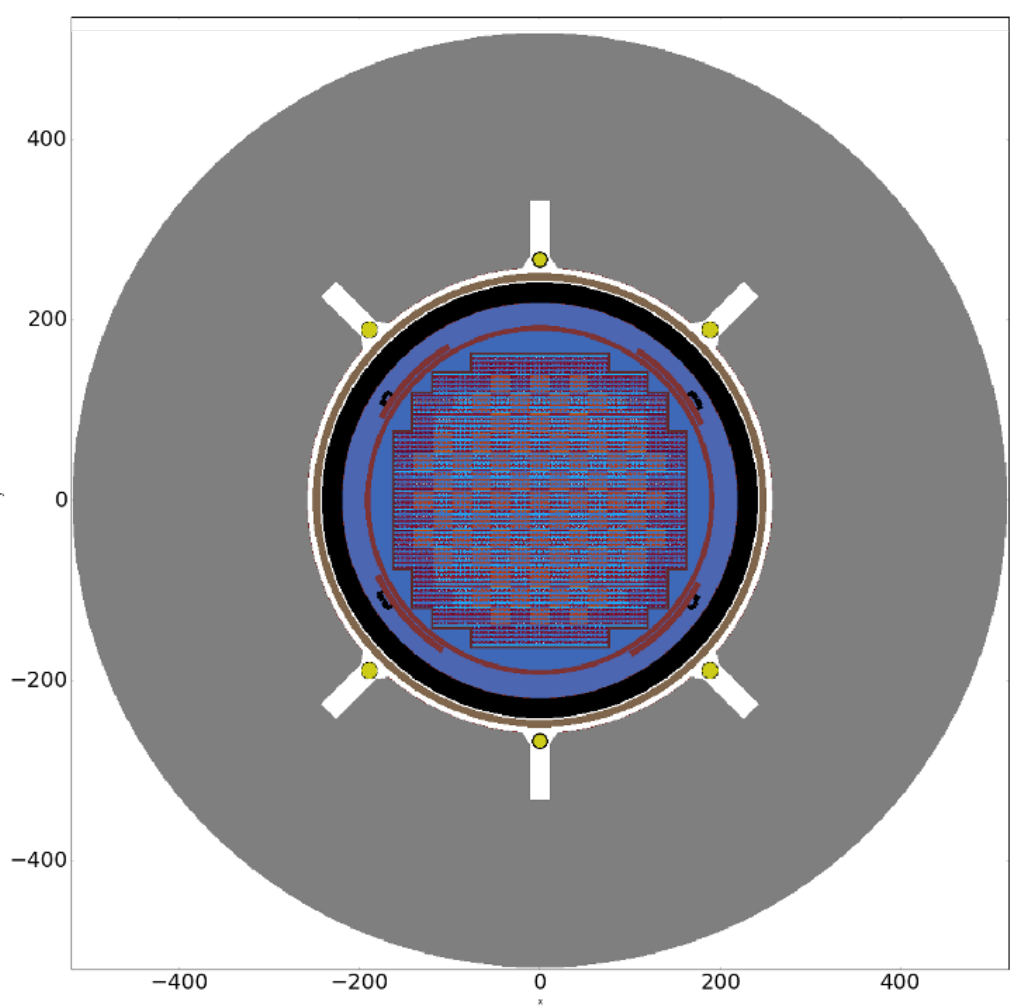

Figure 4. Full core model. 


\subsection{SETTING UP THE OMNIBUS EX-CORE GEOMETRY}

As mentioned earlier, the effort required to set up the ex-core input depends on the complexity of the ex-core region's geometry that the user wants to model. In this section, the steps required to set up the ex-core geometry and any potential user pitfalls are discussed.

Shift does not support rotational symmetry through VERA: it only supports mirror symmetry. Therefore, any VERA input with rotational symmetry must be unfolded manually by the user in the input before running VERA for ex-core calculations. Another quirk to note is that MPACT and Shift model different quadrants when running quarter core problems. This can be a major source of user input error if the user adds features to the ex-core geometry when running a quarter core model with VERA, especially if the ex-core features are not symmetrical. The difference in these quadrants being modeled is shown in Figure 5.

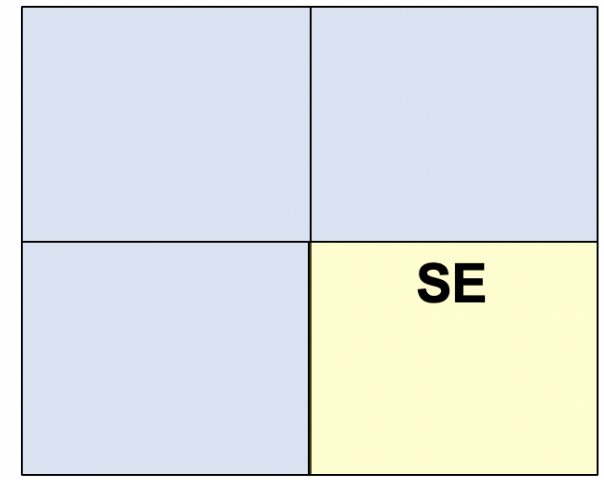

(a) MPACT quadrant

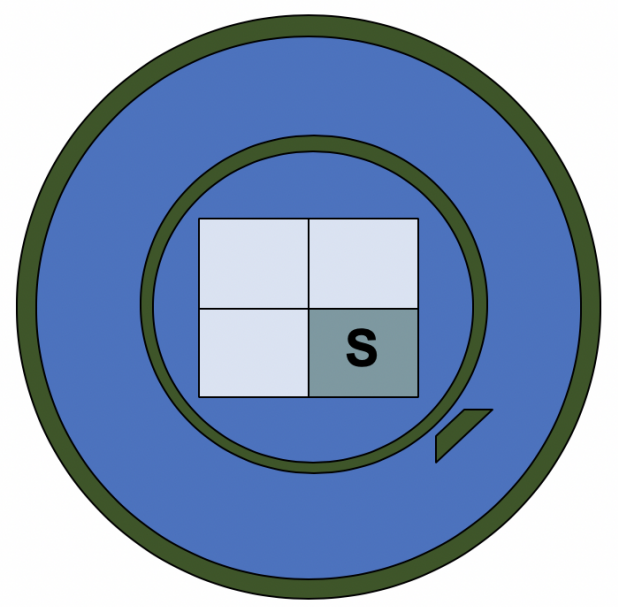

(c) Location of ex-core feature in MPACT quadrant

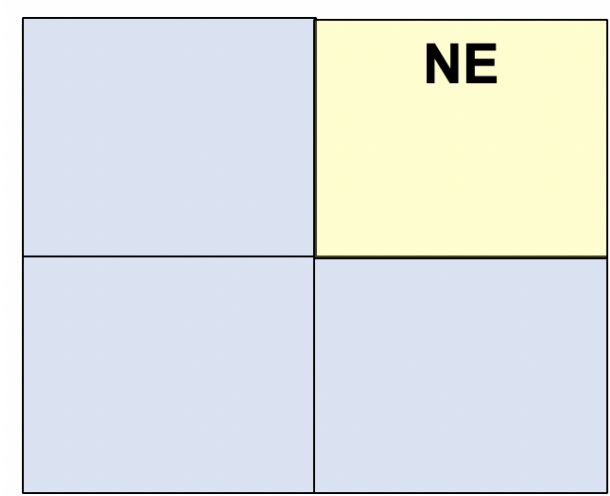

(b) Shift quadrant

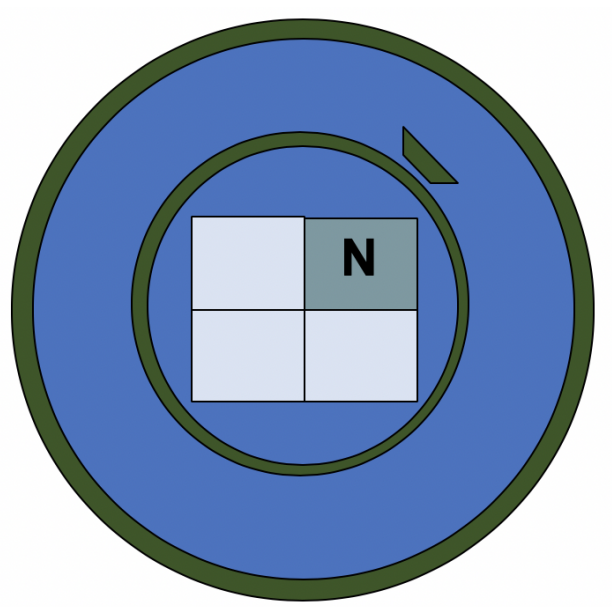

(d) Location of ex-core feature in Shift quadrant

Figure 5. Quarter core quadrants modeled with MPACT and Shift for quarter core problems.

An Omnibus input file requires several blocks of input (Listing 4). Detailed information regarding the set up of Omnibus inputs can be found in the Exnihilo manual (Johnson et al. [2020]). However, the process to set up an Omnibus GG input is revisited here for the purposes of setting up an input for VERA ex-core calculations. 
The input always starts with the [GEOMETRY] block, which tells Omnibus that the input following this block of text will be geometry related. The global parameter is followed by a short string describing the type of geometry being set up in the input file. Next, the user must provide one standard line of input, [UNIVERSE=core reactor], that incorporates the VERA core geometry within the Omnibus input.

Once the VERA core geometry has been incorporated, the boundaries are defined for the entire ex-core geometry. A boundary box is sufficient in most cases, and this bounding box is defined using the cuboid shape in Omnibus. This boundary box must be a cuboid and cannot be another shape because Shift in VERA cannot apply a boundary condition to any other shapes.

Once the boundary is defined, the shapes within the excore universe are defined: coupons, detectors etc. A complete list of shapes and their definitions are inluded in the Exnihilo manual (Johnson et al. [2020]). Table 2 summarizes the shapes available through the Omnibus input format.

Table 2. OMNIBUS shapes

\begin{tabular}{cc}
\hline [SHAPE $=$ TYPE] & DESCRIPTION \\
\hline CUBOID/BOX & Box \\
SPHERE & Sphere \\
CYL & Cylinder \\
CYLSEGMENT/PAD & Cylindrical segment \\
RING/CYLSHELL & Cylindrical shell \\
PRISM & Regular prism \\
SLAB & Infinite slab \\
PLANE & Infinite half-space \\
WEDGE & Wedge \\
CONE & Cone \\
ELLIPSOID & Ellipsoid \\
HOPPER & Hopper \\
RIGHTHET & Right tetrahedron \\
TRIPRISM & Triangular prism \\
ECYLINDER & Elliptical dodecahedron \\
RHOMBDOD & Rhombic dodecahedron \\
PPIPED & Parallelepiped \\
QUADRIC & General quadric \\
\hline
\end{tabular}

After the shapes are defined, holes must be defined to incorporate the core universe from the VERA input or to include any detectors that might have been set up as an independent universe. These holes contain independent universes defined within the global geometry.

The cell definitions follow the hole definitions. All cells can be defined using combinatorial geometry. More examples of this are presented in subsequent sections. The user must bear in mind that there are two "cutoff" points in the radial direction for importing the geometry from the VERA input. The user has the choice of importing the geometry up to (1) the outer radius of the barrel, or (2) the outer radius of the pressure vessel, and then modeling everything beyond that point in the Omnibus ex-core file. For example, the user might consider importing up to the outer radius of the barrel from the VERA input and then modeling everything beyond it in the Omnibus ex-core file to model the coupons and surveillance capsules. In another scenario, a user might want to import everything up to the outer radius of the pressure vessel from the VERA input and then add features beyond the vessel such as a bioshield or ex-core detectors in the Omnibus ex-core file. In the axial direction, the user must ensure that the extents in the z-direction for 
the boundary box extend at least from the bottom of the lower core plate to the top of the upper core plate. Note that all the regions within the boundary box must be defined. A simple Omnibus ex-core input is presented in Section 5.2, but the main pieces of an Omnibus ex-core input are shown below. The [COMP], [RESPONSE] and [TALLY] blocks will be discussed in more detail in the following subsections.

Listing 4. Blocks of input in Omnibus ex-core file.

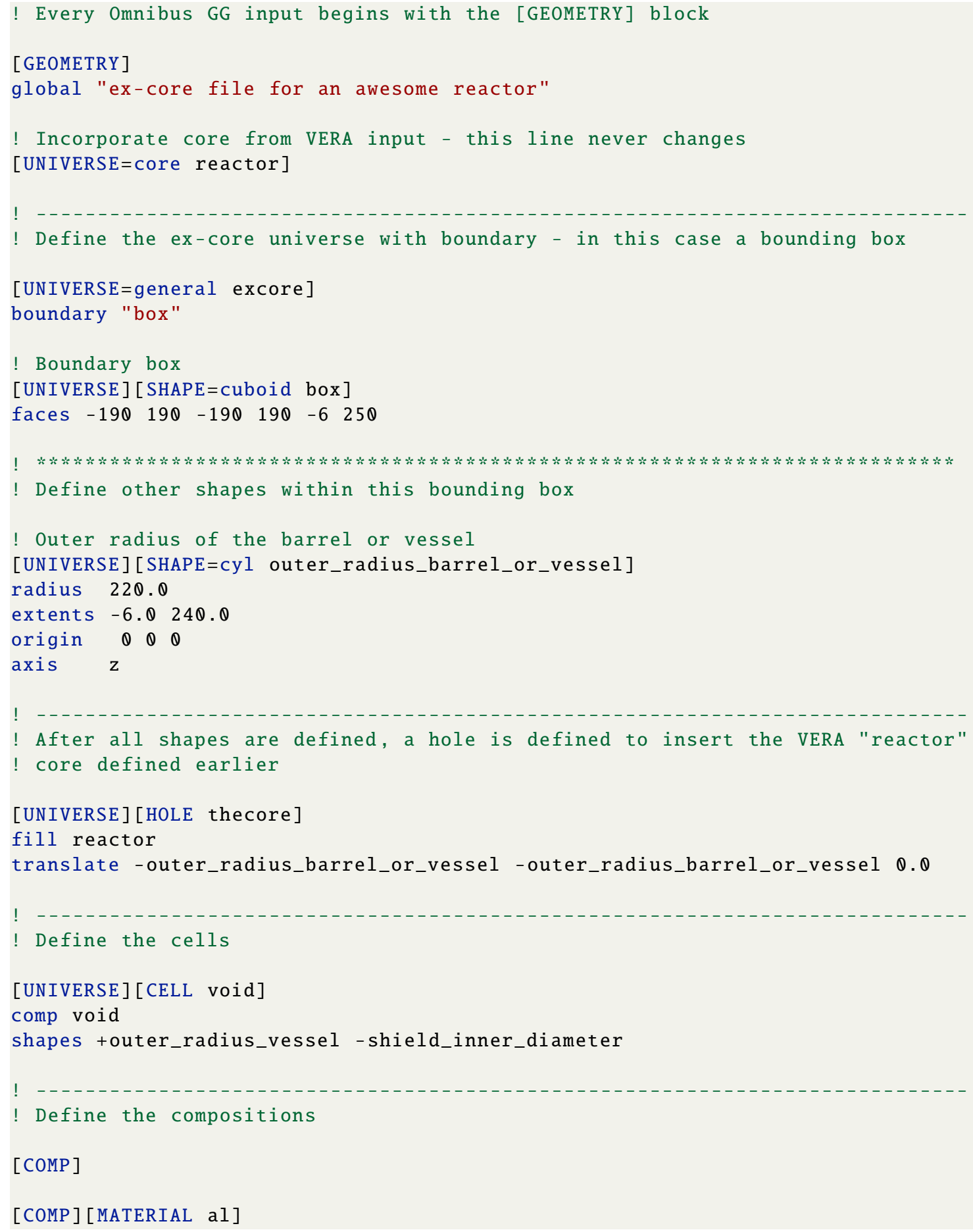




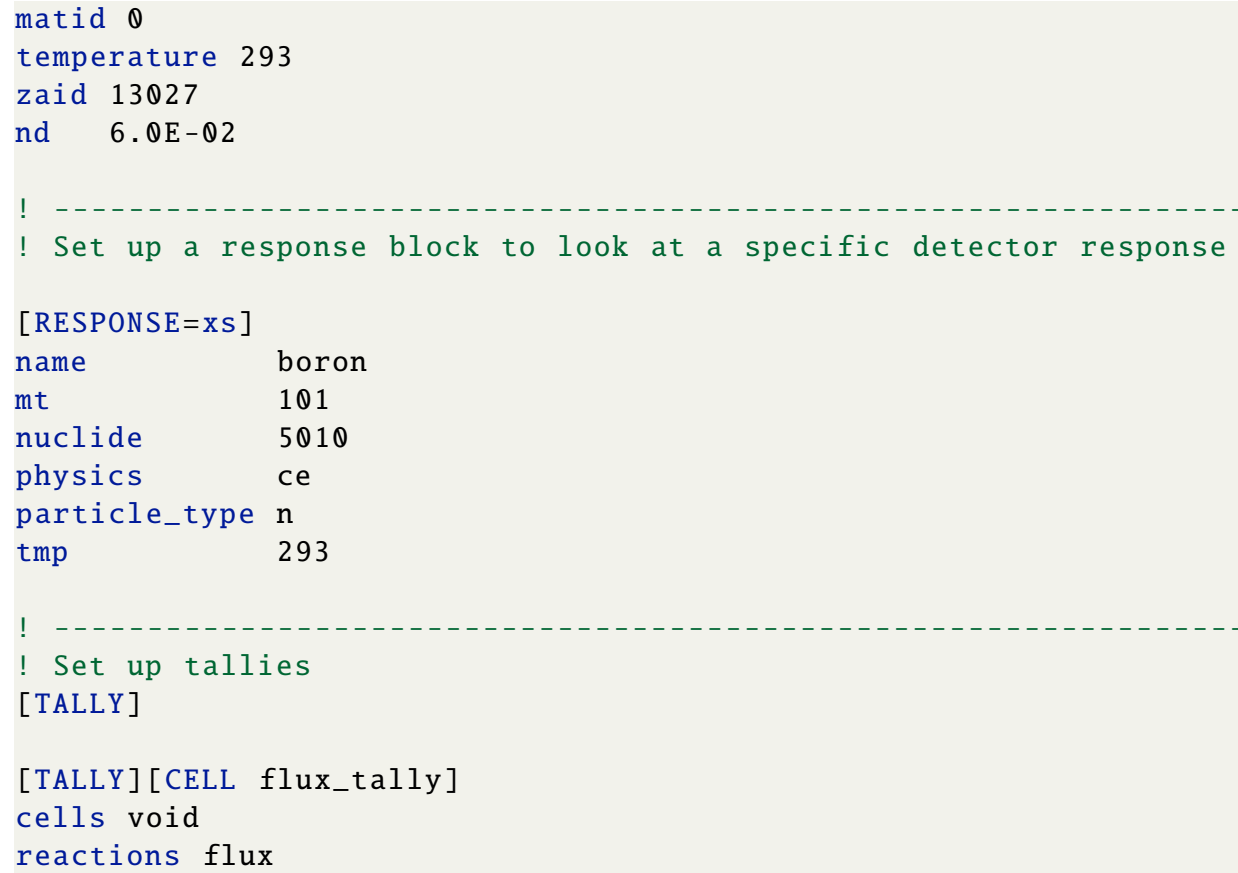

\subsection{A SIMPLE EX-CORE INPUT}

An example of a simple input for an SMR ex-core region as shown in Figure 6 is defined in this section. In this example (Listing 5), everything up to the outer radius of the vessel is pulled in from the VERA input. Only a biological shield is defined outside the vessel using the ex-core input shown below.

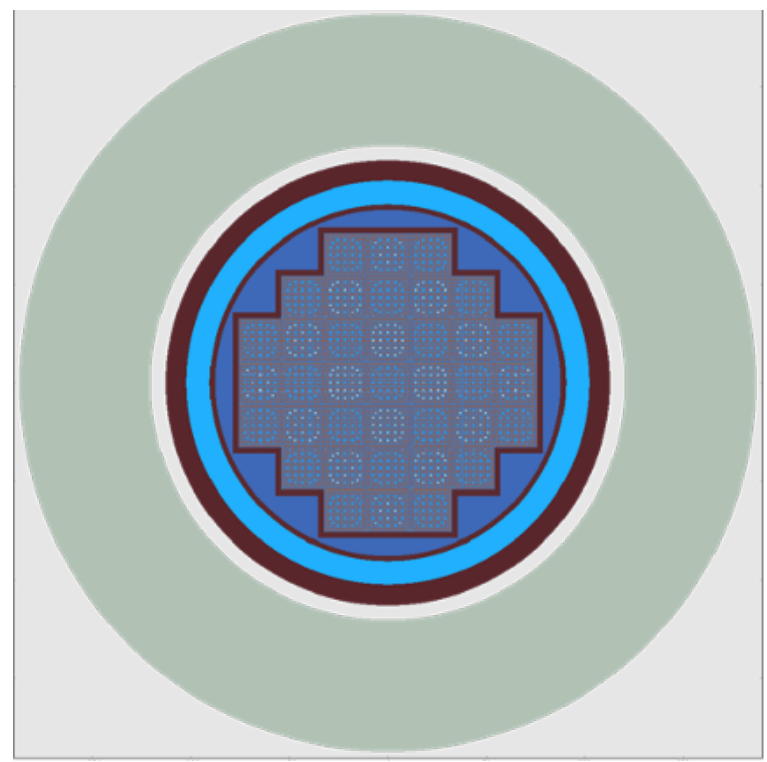

Figure 6. Simple ex-core region for an SMR.

In this input, the global "excore" geometry is defined. Next, the general universe is defined with a 
boundary box that encompasses the entire geometry. Following this definition, the shapes are defined for the bioshield. Note that all geometry up to the outer radius of the vessel is being pulled from the VERA input. Therefore, no additional geometry definitions within the vessel region need to be defined in the Omnibus input file. The HOLE is defined next to integrate the VERA core geometry with the excore universe defined here. Since the VERA and Omnibus origins are not the same, they must be aligned and this alignment is achieved with a translate parameter. This is based on the outer radius of the vessel in this problem and is defined as follows:

translate -outer_radius_of_the_vessel -outer_radius_of_the_vessel 0.0

The holes must be defined after the shape definitions, and this order matters in Omnibus GG. The [CELL] block is defined next using the shapes defined earlier. Any region lying within the surface or has a negative sense to a plane is defined by a negative (-) sign and any region lying outside a surface or has a positive sense to a plane is defined by a positive $(+)$ sign. Next, the compositions and tallies are defined using the [COMP] and [TALLY] blocks. An example for a cell flux tally in the bioshield region is shown. The user can define as many neutron energy bins as desired if they want to tally binned flux; otherwise, the user can omit this line to tally the total flux in the cell.

\section{Listing 5. Simple ex-core file.}

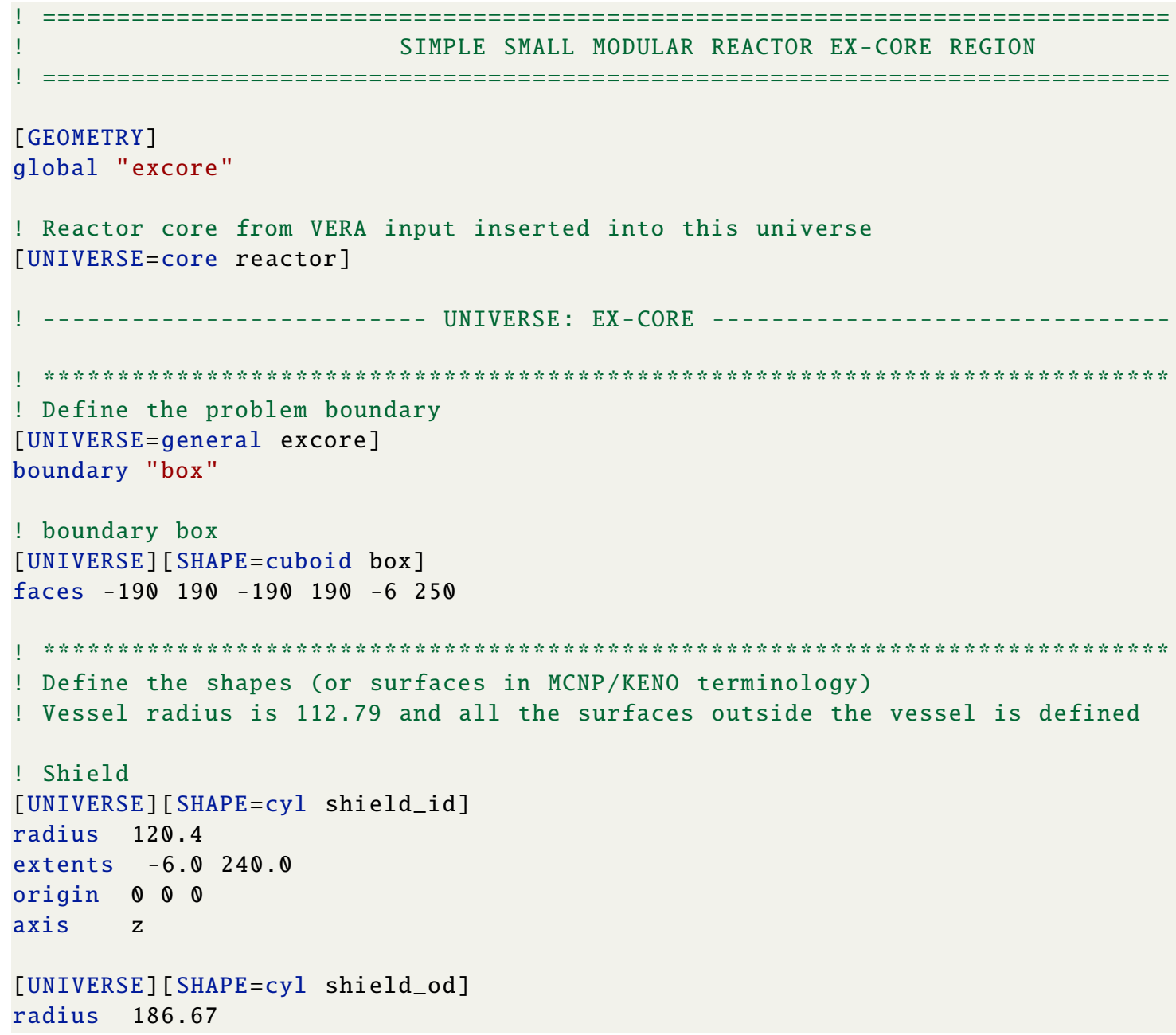




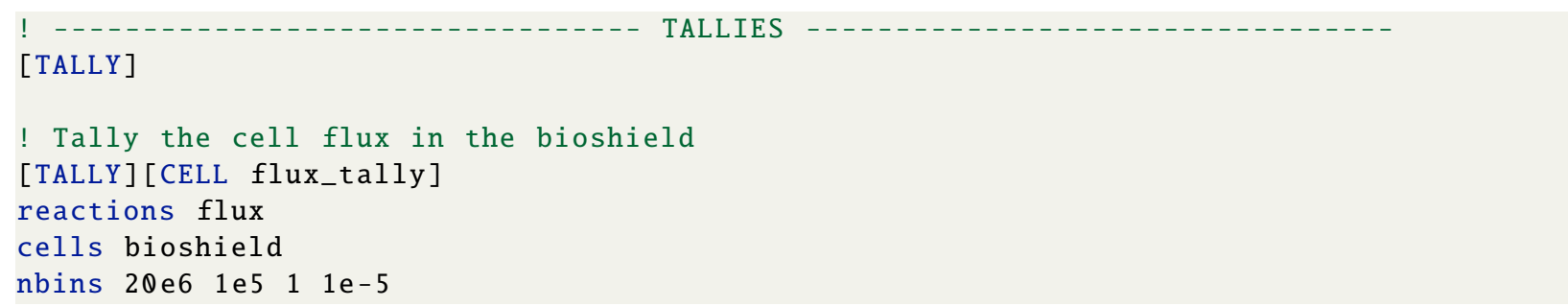

\subsection{SMR EX-CORE INPUT WITH A DETECTOR}

An example of a slightly more complex ex-core input for a small modular reactor (SMR) shown in Figure 7 is defined in this section. A detector well is modeled in the ex-core region in this model.

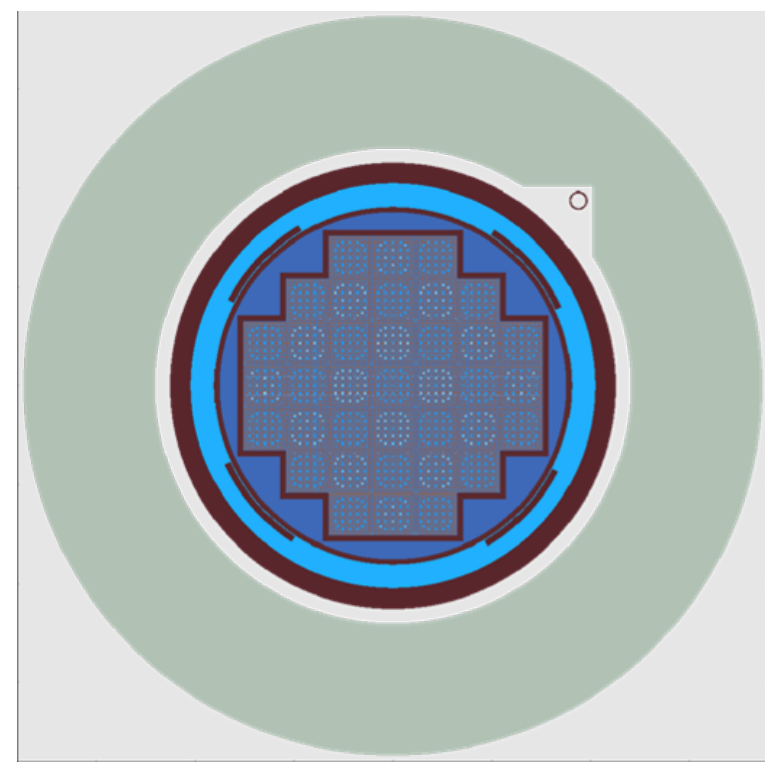

Figure 7. An SMR with an ex-core detector.

See Listing 6 for the Omnibus input. The first step in setting up the ex-core geometry is to define the [GEOMETRY] block and to also integrate the VERA core geometry with the ex-core geometry using the [UNIVERSE=core reactor] block. Next, a separate universe that defines the detector region in the northeast quadrant is set up. This universe is called det_cutout and has a boundary shape called shield_id. Under the SHAPE (or surface) definitions, it can be seen that shield_id is a ring shape, and the detector lies within this ring. The ring's inner and outer radii are the inner and outer radii of the bioshield. The detector well region is defined by the cuboid shape, which is cut by the ring, giving it the detector well shape. The detector is split into top and bottom halves, and a pipe surrounds the detector that extends the entire length of the top and bottom halves. After the shapes are defined in the det_cutout universe, the cells are defined. Finally, the hole_boundary for the det_cutout universe must be defined. The universe lies within the shield_id boundary.

After the det_cutout universe has been defined, the excore universe is outlined. This universe contains a boundary box shape that encompasses the entire geometry. In this example, the geometry up to the outer 
radius of the barrel from the VERA input is integrated into this ex-core file. All regions beyond the barrel are defined in the excore universe. This means that the pads, reactor pressure vessel liner, and vessel in the ex-core file must be redefined in the ex-core file. This gives the user the flexibility to add surveillance capsules off the pads or within the vessel itself, or it allows the user to expand the ex-core region beyond the vessel to add the bioshield and to add a detector, as in this case.

After the boundary for the problem has been defined, the next step is to define all the shapes to be represented in the ex-core geometry. After the shape definitions, the HOLES are defined to integrate the universes defined earlier within the excore universe. In this example, the reactor universe represent the VERA geometry and the detector_cutout universe representing the detector well and the detector are inserted within the excore universe. The reactor universe is translated within thecore hole because the origin of the VERA geometry and the Omnibus geometry must align. This translation is based on the outer radius of the barrel for this problem and is defined as follows:

translate -outer_radius_of_the_barrel -outer_radius_of_the_barrel 0.0

The holes must be defined after the shape definitions, and this order matters in Omnibus GG. Next, the cells are defined. All regions inside a surface is defined by the minus (-) symbol, and all regions outside a surface are defined by the plus (+) symbol. For example, the moderator region is defined by all of the region outside the VERA geometry that is defined by +thecore, inside the reactor vessel liner (-rpv_liner), and outside the surfaces defining the pads (+pad_ne +pad_nw +pad_sw +pad_se). The user must keep in mind that all the regions extending up to the problem boundary, which are defined as box in this case, must be defined using cells.

After all the shapes have been defined, the next block is the [COMP] blocks in which the compositions are defined. Note that these compositions are referenced in each cell that was defined earlier to specify the material in that cell.

The [RESPONSE] blocks are defined next, and here, any specific types of responses related to dose or reaction rates are specified. The example here sets up a response for fission reactions in ${ }^{235} \mathrm{U}$. The user can also specify the associated MT values from the ENDF files here. This response can then be used within the [TALLY] block that is set up next to calculate the detector response. Different types of [RESPONSE] blocks can be found in the Johnson et al. [2020].

\section{Listing 6. Ex-core file with bioshield and detector.}

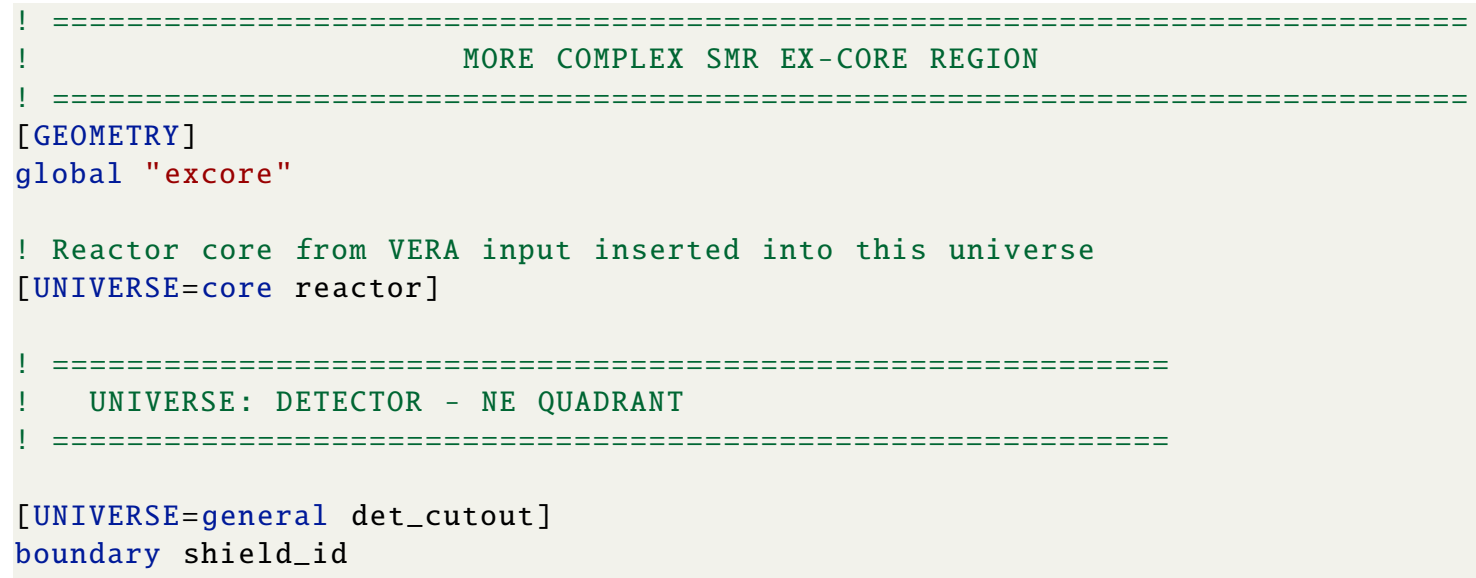




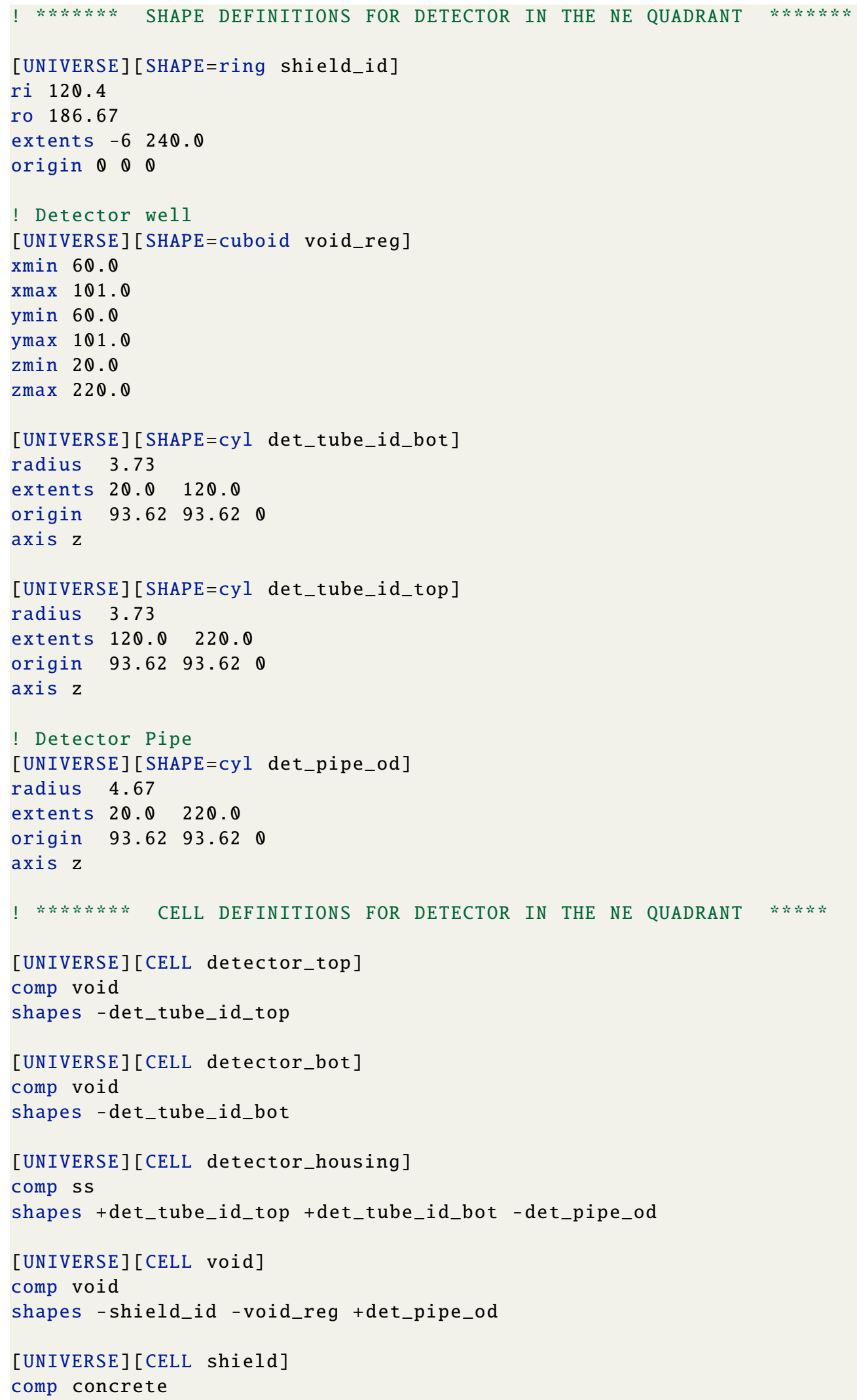




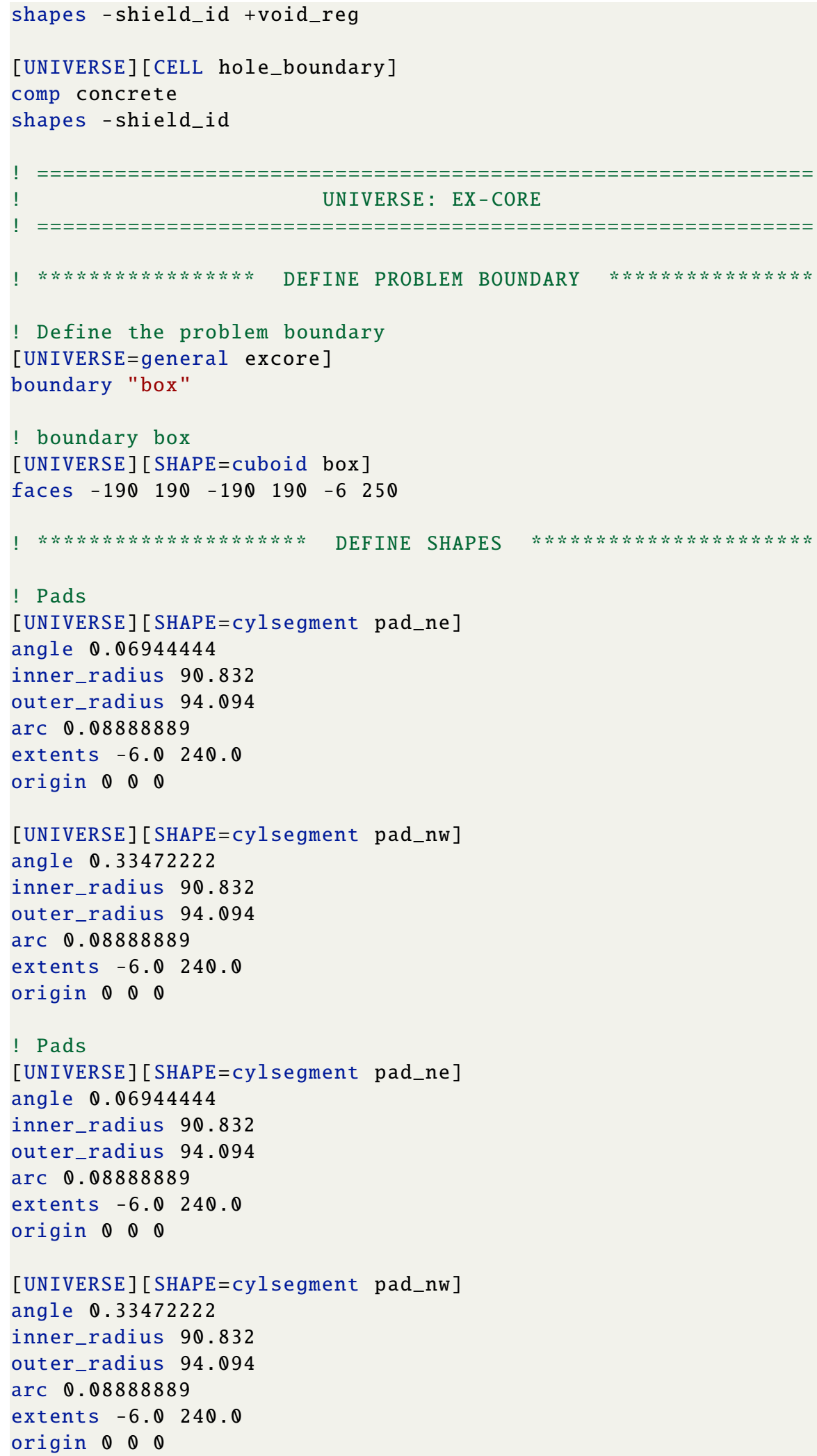




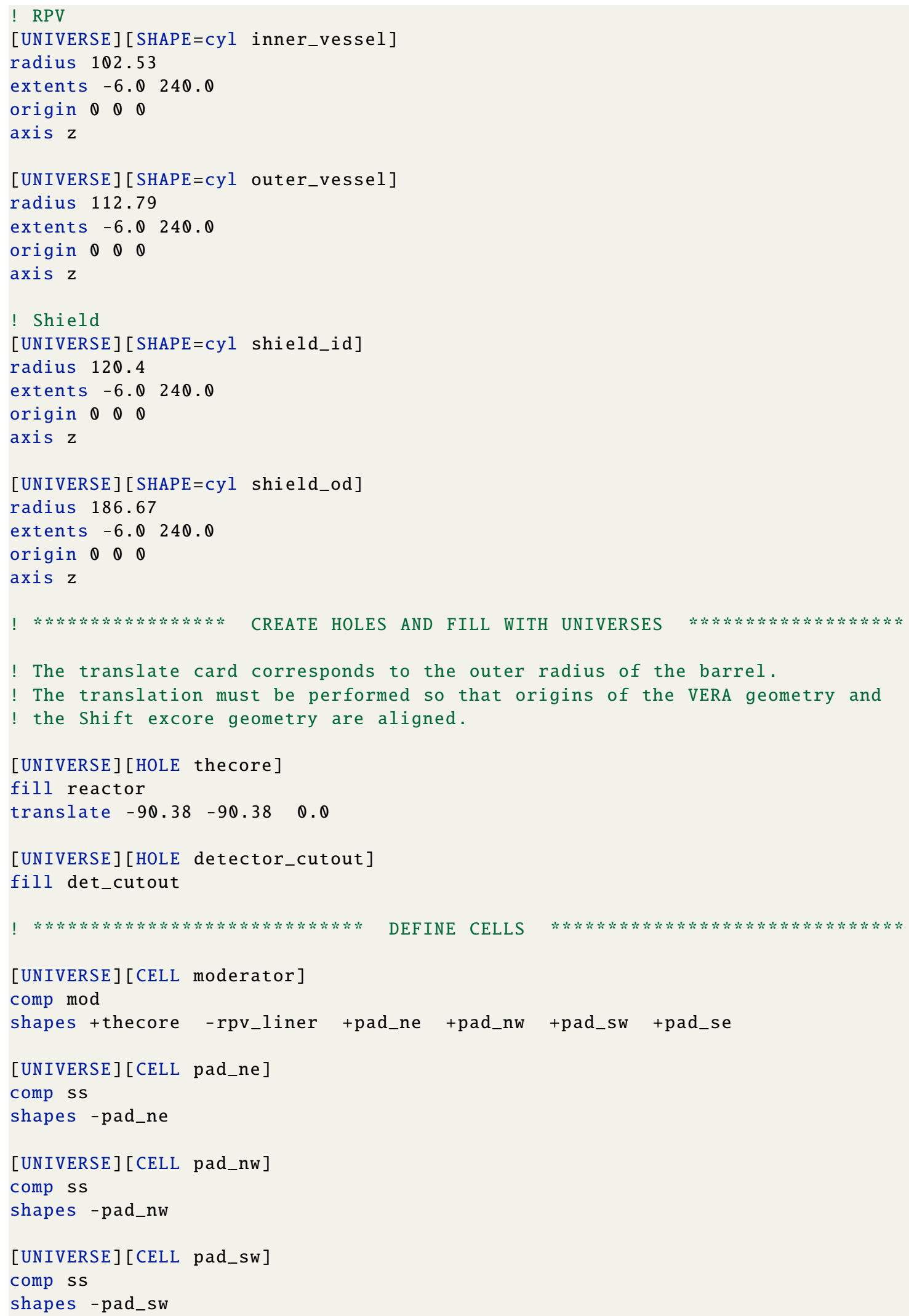




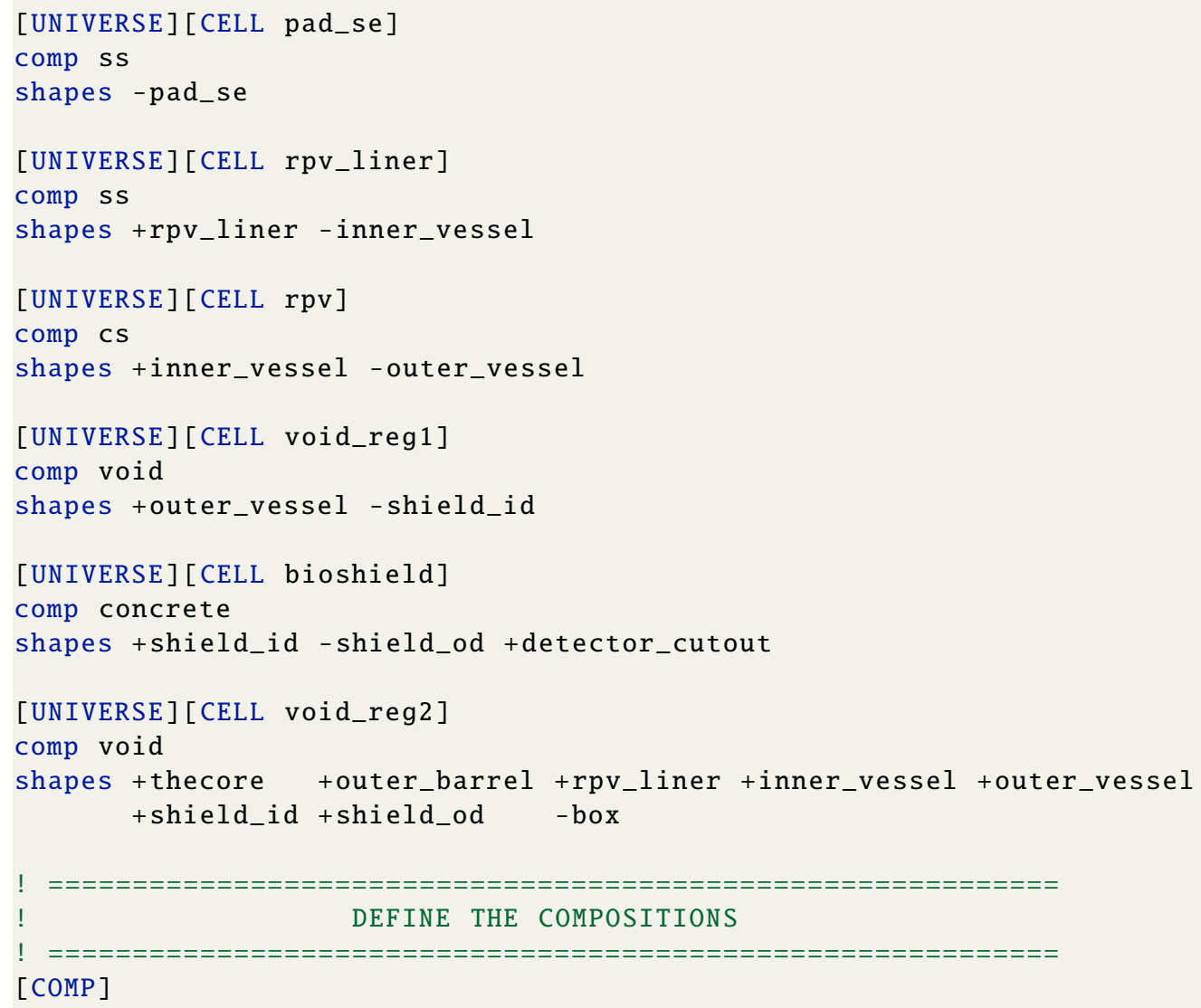




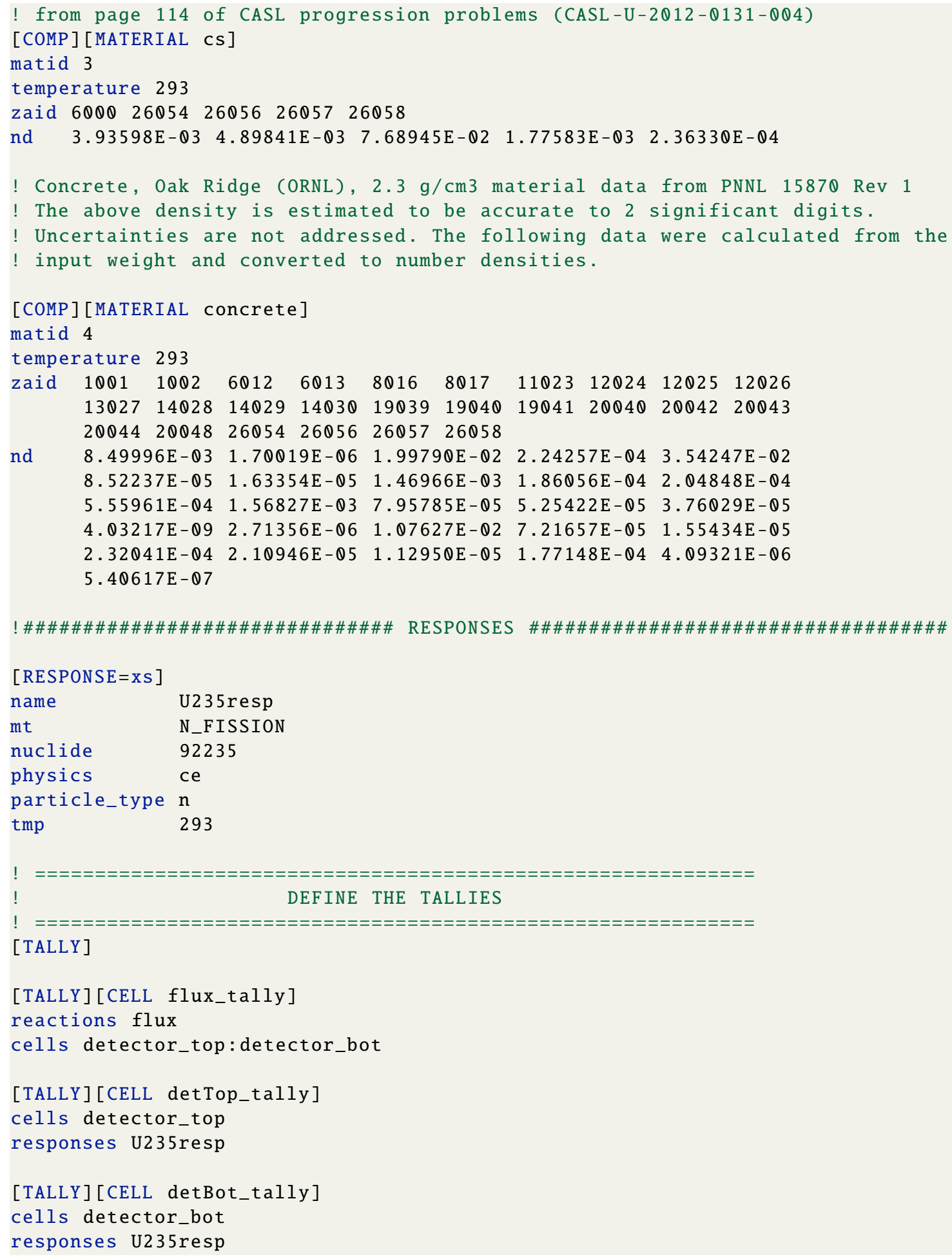




\subsection{AUTOMATED GENERATION OF THE BIOSHIELD AND EX-CORE DETECTORS}

VERA can generate an automated detector input, which is in an Omnibus ex-core file format (*. omn), so that the user does not have to manually create an ex-core input file from scratch. This is a good way to start learning how to generate an ex-core file for a user unfamiliar with how to set up an Omnibus GG input.

The [CORE] block in the VERA input needs some additional user input regarding the bioshield and the detector (see Listing 7). Let us assume in this example that the height of the active core region (from the top of the bottom core plate to the bottom of the upper core plate) is $240 \mathrm{~cm}$. Therefore, the mid-plane of the active core region is at $\mathrm{z}=120 \mathrm{~cm}$. A bioshield is first set up, and it extends radially from $120.0 \mathrm{~cm}$ to $250 \mathrm{~cm}$. Following this definition, there are four types of detectors that are set up in six different locations.

\section{Listing 7. VERA input file for automated detector generation.}

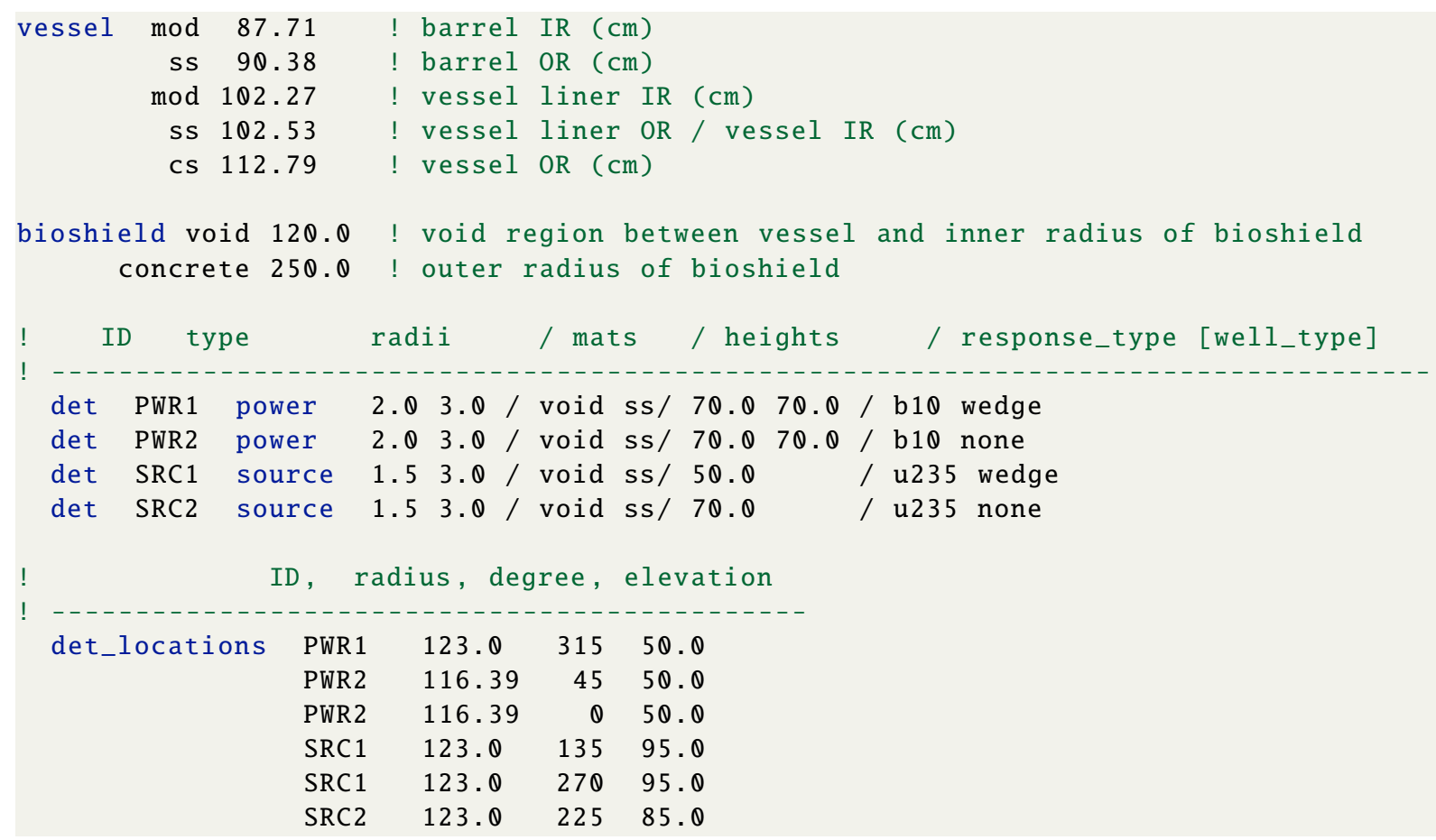

The four types of detectors that are set up are addressed first. The user must first up a detector using the parameter det, and then the user must provide the detector ID, such as PWR1. The detector ID can be any string that the user would like to specify. The detector type is defined after the detector ID. The two types of detectors are power for power range detectors and source for source range detectors. Next, the user has to enter the radius of the detector followed by the radius of the metal casing around the detector. Note that the user can enter as many concentric rings within the detector as needed. For example, the user can specify an inner void region followed by an aluminum casing, and then another void region and an outer steel casing. Once the radii have been defined, the user can specify the materials in each region. In this case, the inner region is void, and the outer region is ss. The default compositions available for the automated detector set up are provided in the ex-core manual Pandya et al. [2020], and is also provided in Listing 8 for completeness. 


\section{Listing 8. Default materials in automatically generated ex-core file.}

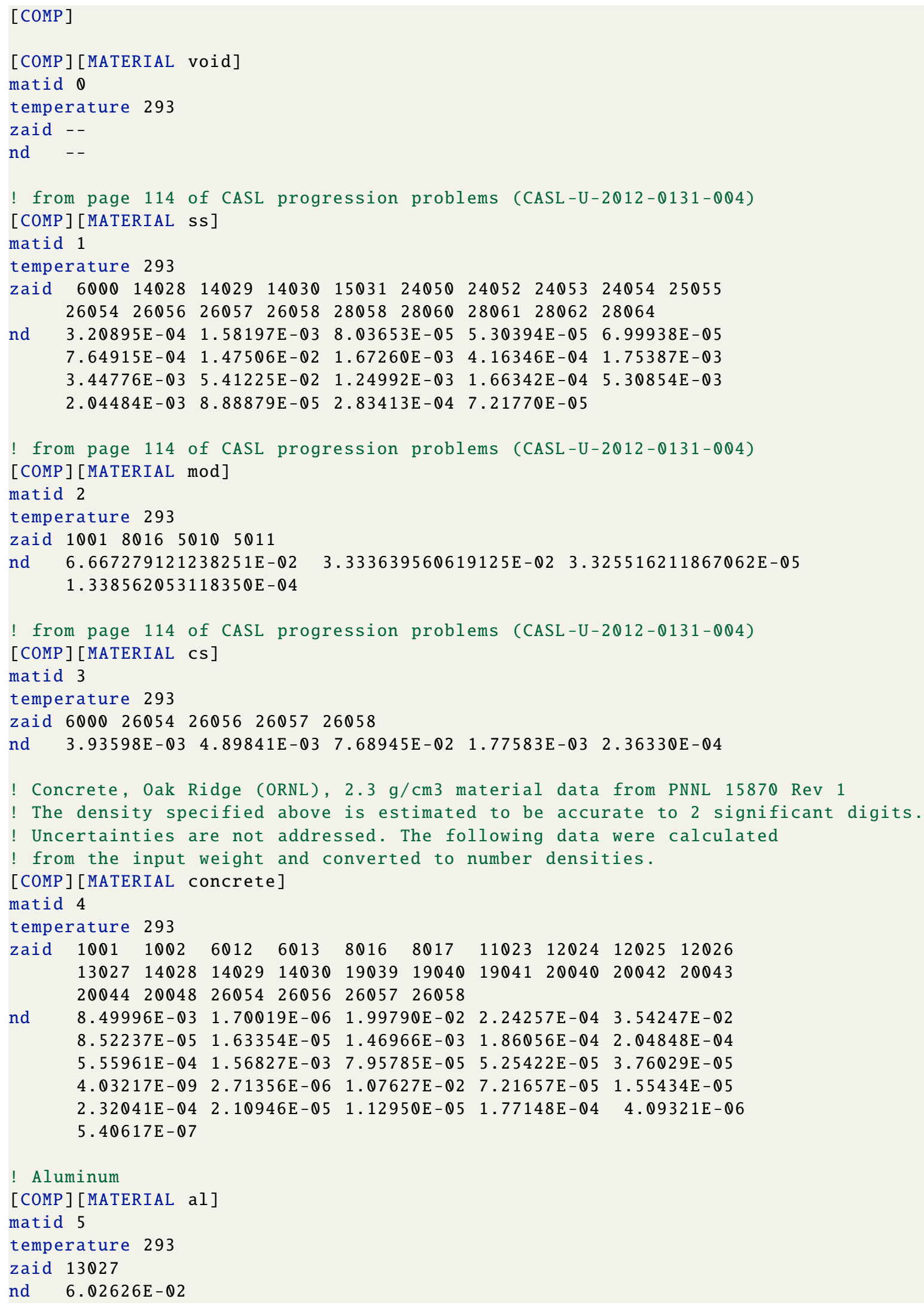


After the material has been defined, the user must specify the height of the detector. The height can be divided into two axial regions with top and bottom halves, as in the case of PWR1 and PWR2, or the detector can have one axial region, as in the case of SRC1 and SRC2. After the detector height is specified, the response type must be specified. The detector can be set up with absorption reactions in ${ }^{10} \mathrm{~B}$ or fission reactions in ${ }^{235} \mathrm{U}$. Finally, the detector well type is specified. A wedge-shaped well can be specified, or the detector can be defined without a well.

Then the detector locations for each of the detector types are provided with radius and degrees. Note that when defining the degrees, the user must bear in mind that 0 to $90^{\circ}$ refers to the southeast quadrant, 90 to $180^{\circ}$ is the southwest quadrant, 180 to $270^{\circ}$ is the northwest quadrant, and 270 to $360^{\circ}$ refers to the northeast quadrant. The elevation of the detector refers to the distance from $\mathrm{z}=0 \mathrm{~cm}$, which is the top of the bottom core plate to the bottom of the detector if there is only one axial region in the detector. Or, if there are two axial regions, then it refers to the distance from the top of the bottom core plate to the bottom of the detector located in the lowest axial region. In this example, the mid-plane of the core is at $\mathrm{z}=120 \mathrm{~cm}$, so for the PWR1 and PWR2 detectors, the elevation is $50 \mathrm{~cm}(120$ minus $70 \mathrm{~cm})$. The elevation for SRC1 is $95 \mathrm{~cm}$ $(120$ minus $25 \mathrm{~cm})$, and the elevation for SRC2 is $85 \mathrm{~cm}(120$ minus $35 \mathrm{~cm})$, which makes them axisymmetric about the core mid-plane. A plot of the detectors is shown in Figure 8.

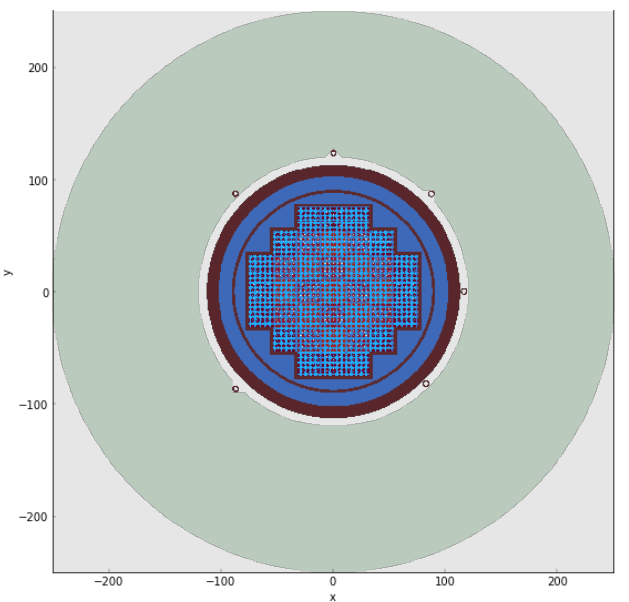

(a) Detectors and bioshield

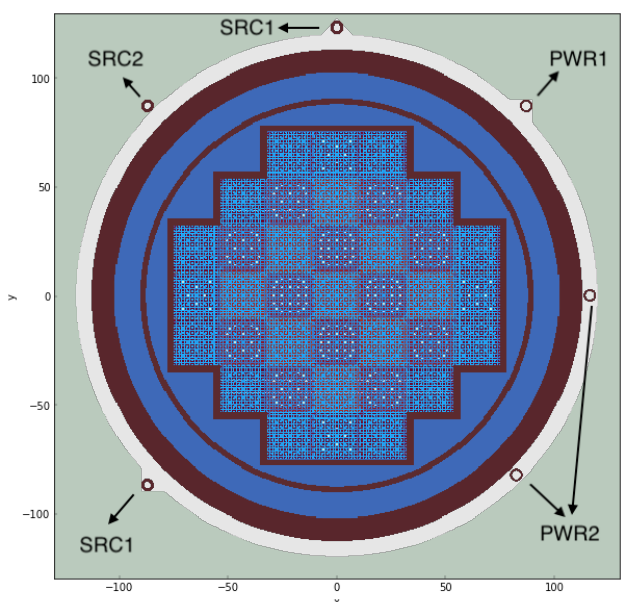

(b) Close-up view of the detectors

Figure 8. Automatically generated detectors and bioshield.

The next step is to ensure that in the [SHIFT] block of the VERA input file, the parameter vera_pressure_vessel is set to true, and the core_translate parameter corresponds to the outer radius of the reactor pressure vessel and is set to the following:

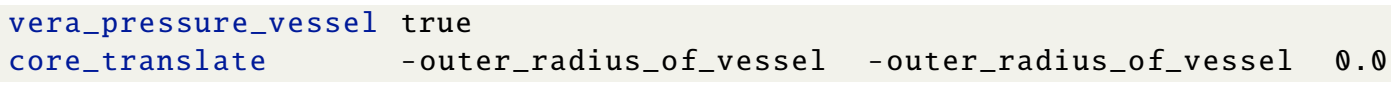

More details regarding the set up of the [SHIFT] block are provided in Section 5.5. Once these parameters have been added to the Shift block, if the user does not intend to change the default materials or their temperatures, then VERA can be run using verarun. However, if the user would like to update the materials and temperatures, then there are two options: (1) submit a job using verarun vera_input_file.inp command and then immediately kill the job that was just submitted using the command qdel jobid, or (2) run the react $2 \mathrm{xml}$ executable by typing the following into the terminal: 
An ex-core vera_input_filename.omn file will be output to the working directory. The user can then scroll down to the [COMP] block to make any changes to the material, number densities, or the temperatures for the appropriate detectors. The detector ID is provided in the comments within the input to make it is easier for the user to identify the cells associated with a detector. By scrolling down to the [TALLY] block, the user will see a list of tallies associated with the detectors set up in the VERA input. For this example, see Listing 9 for the tallies automatically generated in the ex-core input file.

\section{Listing 9. [TALLY] block automatically generated in the ex-core file.}

\section{[TALLY]}

[TALLY] [CELL detector_Q_o]

cells detector_Q_o

responses b10

[TALLY] [CELL detector_Q_2]

cells detector_0_2

responses b10

[TALLY] [CELL detector_1_Q]

cells detector_1_0

responses b10

[TALLY] [CELL detector_1_2]

cells detector_1_2

responses b10

[TALLY] [CELL detector_2_o]

cells detector_2_o

responses b10

[TALLY][CELL detector_2_2]

cells detector_2_2

responses b10

[TALLY][CELL detector_3_Q]

cells detector_3_0

responses u235

[TALLY] [CELL detector_4_Q]

cells detector_4_0

responses u235

[TALLY][CELL detector_5_0]

cells detector_5_0

responses u235

detector_Q_Q 0 and detector_Q_2 are associated with the lower and upper detector regions of PWR1. detector_1_0 and detector_1_2 are associated with the lower and upper detector regions of PWR2 located at a radius of $116.39 \mathrm{~cm}$ and $45^{\circ}$. detector_1_Q and detector_1_2 are associated with the lower and upper detector regions of PWR2 located at a radius of $116.39 \mathrm{~cm}$ and $0^{\circ}$. Next, detector_3_0 
and detector_4_Q are associated with SRC1 detectors located at a $123 \mathrm{~cm}$ radius and at $135^{\circ}$ and $270^{\circ}$, respectively. Finally, detector_5_0 is associated with the SRC2 detector located at a $123 \mathrm{~cm}$ radius and at $225^{\circ}$.

The next step can be prone to error, so it is highly recommended that this modified * omn file be saved in a separate folder so that it is not accidentally overwritten. Once a backup of this ex-core file has been created, the next step is to run verarun again by typing in the input file name but this time with the *.xml extension.

verarun vera_input_filename.xml

This will bypass the react $2 \mathrm{xml}$ execution, and a new *.omn file with the default materials will not be produced and override the modified *.omn file with the updated materials. After the execution of the *. xml file, open the *. omn file and make sure that the density and temperature changes that were made show up accurately and were not accidentally overwritten. The user can also check* . excore. xml to ensure that the correct compositions and temperatures were indeed used in the calculation.

\subsection{RUNNING VERA WITH AN EX-CORE FILE}

Once the ex-core file has been set up correctly, the [SHIFT] block in the VERA input must be set up correctly to account for the additional ex-core file. See Listing 10 for an example.

\section{Listing 10. [SHIFT] block in VERA input for running ex-core calculations with an ex-core file.}

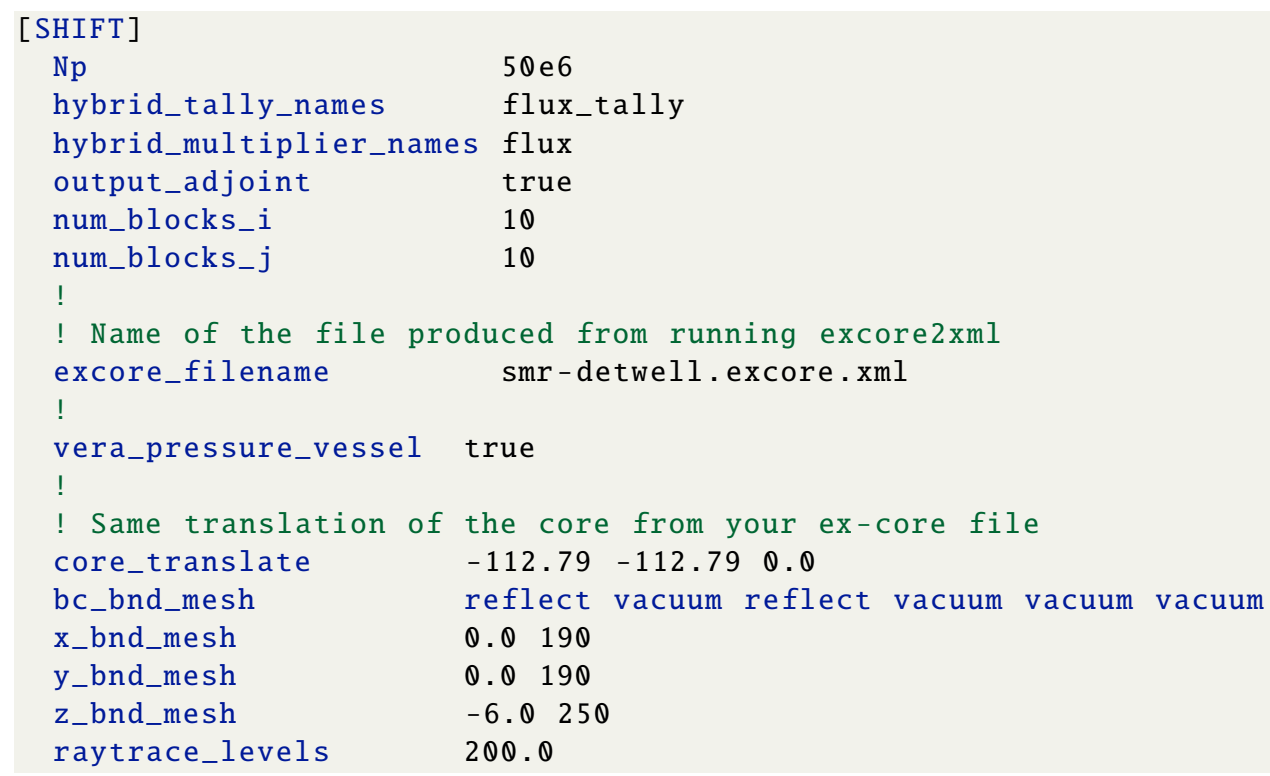

Np represents the total number of particles to be used in the Shift fixed source calculation. hybrid_tally_names represents the name of the tally in the ex-core file that the user would like to optimize in the hybrid calculation. If the user is interested in optimizing for the flux_tally shown in Section 5.3, then that needs to be specified using the hybrid_tally_names. Note that the user can optimize for multiple cell tallies. The hybrid_mulitplier_names should be set to flux since this is the 
only option currently available to the user because Shift integrated in VERA cannot currently optimize for other responses such as dose with CADIS. It can only optimize for flux.

If the user would like to plot the adjoint, then the output_adjoint option should be set to true. The user can also change the default setting of num_blocks_i and num_blocks_j to lower or higher numbers so that the Shift transport calculation runs on a lower or higher number of processors. Note that num_blocks_i multiplied by num_blocks_j is the total number of processors used for the Shift calculation. Next, the user must specify the name of the ex-core file using the excore_filename parameter and append it with the *. excore. xml extension. This definition is necessary because VERA will run a script that converts the ex-core file to an $\mathrm{xml}$ format which is then read by Shift to perform transport on the ex-core geometry.

If the user is integrating all the geometry up to the reactor pressure vessel's outer radius from the VERA input, with the remainder of the geometry outside the pressure vessel defined in the ex-core file, then the user must set the vera_pressure_vessel parameter to true. This is also a requirement for cases run with the automated generation of the detector and bioshield. However, if the user is only integrating the geometry up to the outer radius of the barrel from the VERA input and then defining all the geometry outside the barrel using the ex-core file, then vera_pressure_vessel must be set to false.

The user must also align the origin in the VERA input with the origin in the ex-core file. Note that this was done previously in the ex-core file using the translate card when integrating the VERA geometry using the HOLE. It must be defined again here in the [SHIFT] block, and the translation remains the same. The translation in the VERA input is provided using the core_translate parameter. This is another potential user pitfall.

Next, the boundary conditions must be specified. The bc_bnd_mesh parameter allows the user to specify the boundary conditions along the $-x+x-y+y-z+z$ boundaries, in that order. In this example, the boundary conditions are provided for a quarter core problem, so the boundary conditions are reflect vacuum reflect vacuum vacuum vacuum. Next, the problem boundaries along $\mathrm{x}, \mathrm{y}$ and $\mathrm{z}$ are provided using the x_bnd_mesh, y_bnd_mesh, and z_bnd_mesh parameters. The user may provide the same boundaries that were used for boundary box in the ex-core file here. Note that the user must define the full geometry in the ex-core file even if they are running a quarter core geometry in VERA. Shift performs the fixed-source transport on the full geometry. Finally, the user can specify a z-plane along which a raytrace will be performed for plotting purposes using the raytrace_levels parameter. This will allow the user to produce an image of the VERA geometry integrated with the ex-core geometry. 



\section{PRE- AND POST-PROCESSING INPUTS AND OUTPUTS}

When setting up a geometry, the user should check to ensure that there are no geometry errors. A python script has been set up for this purpose so that Omnibus modules associated with the geometry raytracing can be loaded and then used to produce plots to check for errors.

\subsection{CHECKING FOR GEOMETRY ERRORS DURING EX-CORE INPUT SETUP}

It is important for analysts to view the geometry and check for any geometry errors while setting up the ex-core geometry. Unfortunately, the only way to do this currently is to run a python script that generates plots at different planes.

To run the script, a few changes must be made to the ex-core * omn file from that discussed in previous sections. Listing 11 provides an example showing how to set up an ex-core file for geometry visualization and error checking. First, the [TALLY] and [COMP] blocks at the bottom of the input files must be commented out or removed, and then the user must specify a comp:matid list at the beginning of the ex-core file for each composition specified in the ex-core file (see an example below). Lines that are to be commented out, modified, or added are highlighted in yellow. The first example shown of a simple SMR ex-core file in Section 5.2 is modified here so that plots of the geometry can be obtained to check for any errors before running it with VERA.

Next, the [UNIVERSE=core reactor] block must be commented out, and in its place, a new [UNIVERSE=general reactor] must be defined. This step is necessary because the user is must generate the geometry using the python script before running VERA. Therefore, the code does not have the information required to plot the VERA geometry. In its place, a "fake core" is modeled which models a cylinder up to the outer radius of the barrel or the outer radius of the reactor pressure vessel, depending on the boundary up to which the VERA geometry is integrated. Finally, the user must remove or comment out only the translate card for the [HOLE thecore] since this translate card is only aligning the VERA geometry origin with the Shift ex-core origin.

Listing 11. Revised ex-core file for geometry visualization and error-checking.

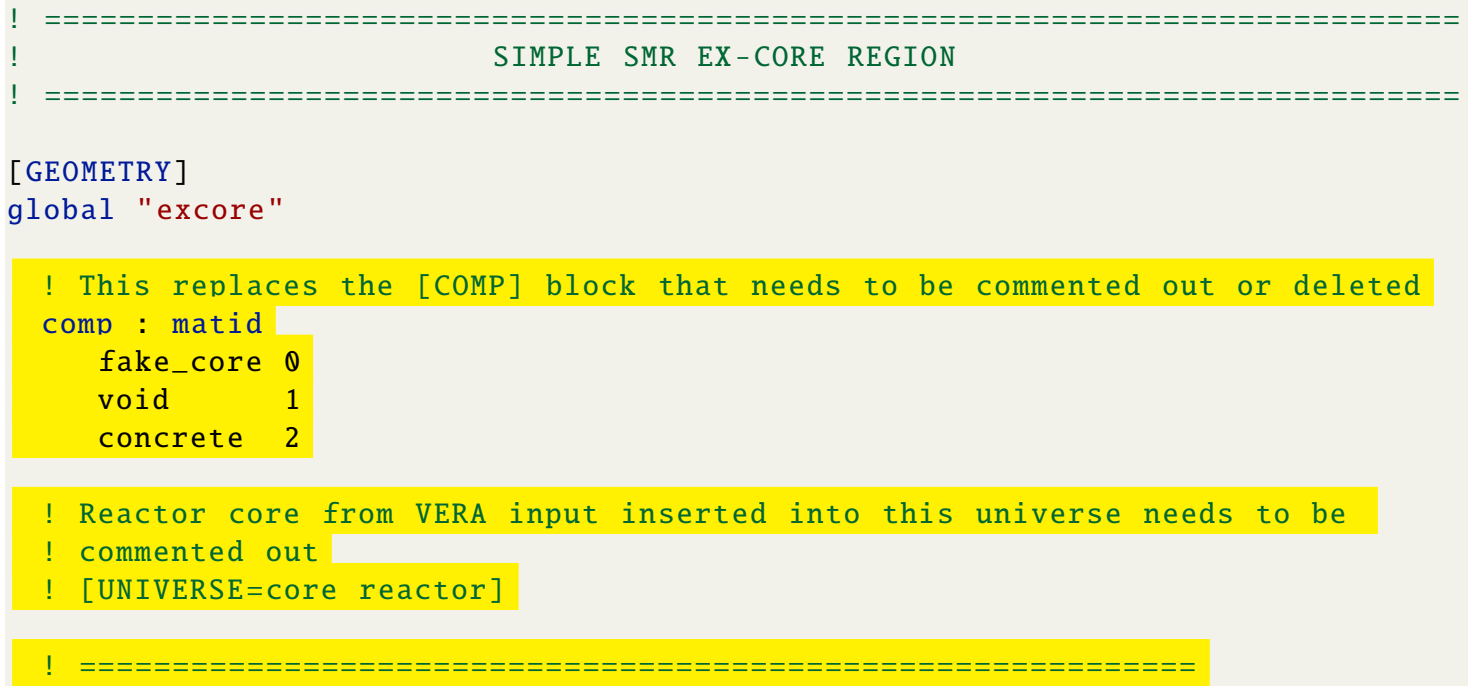

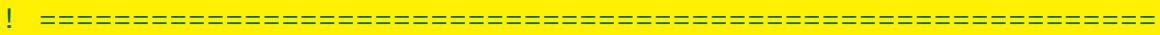




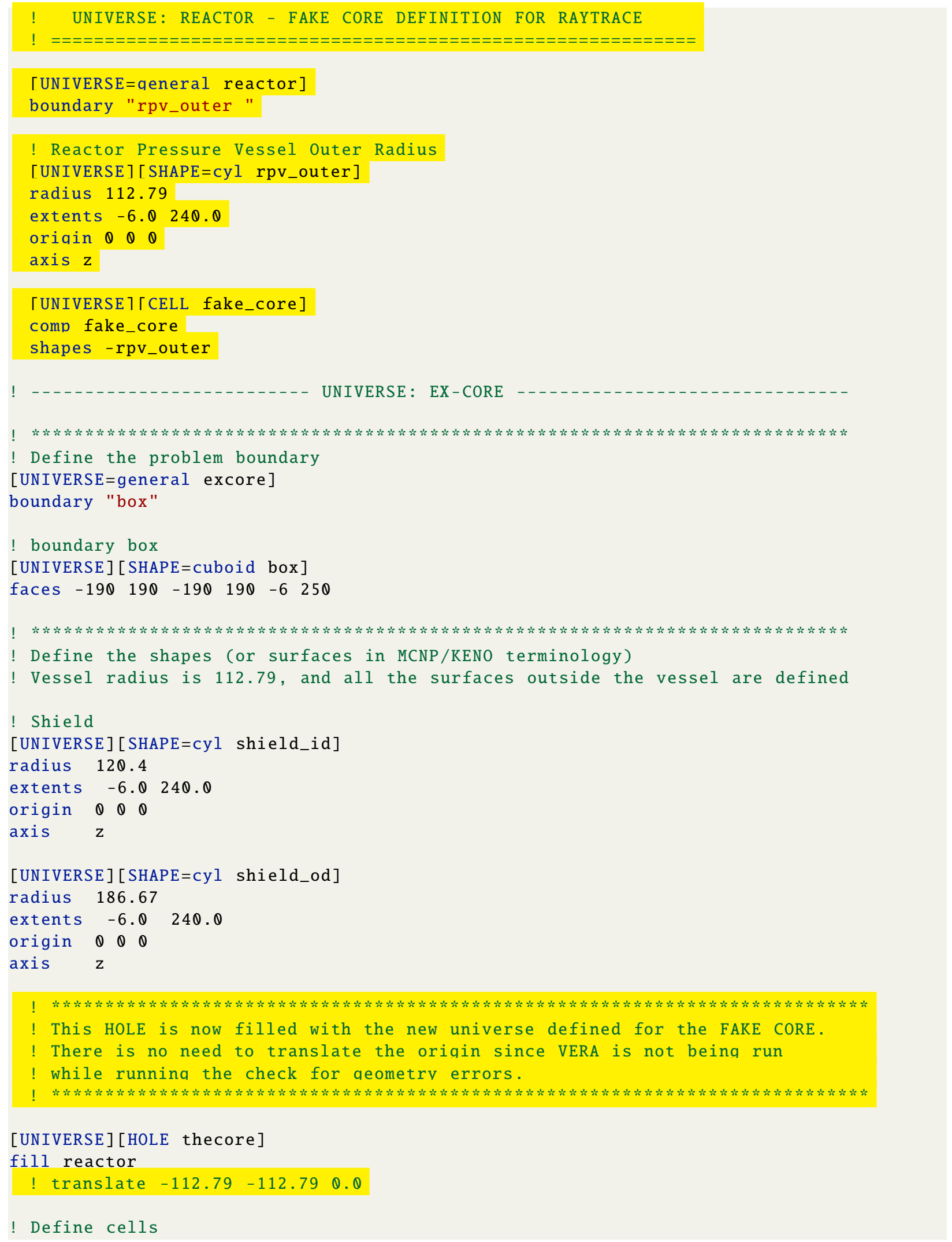




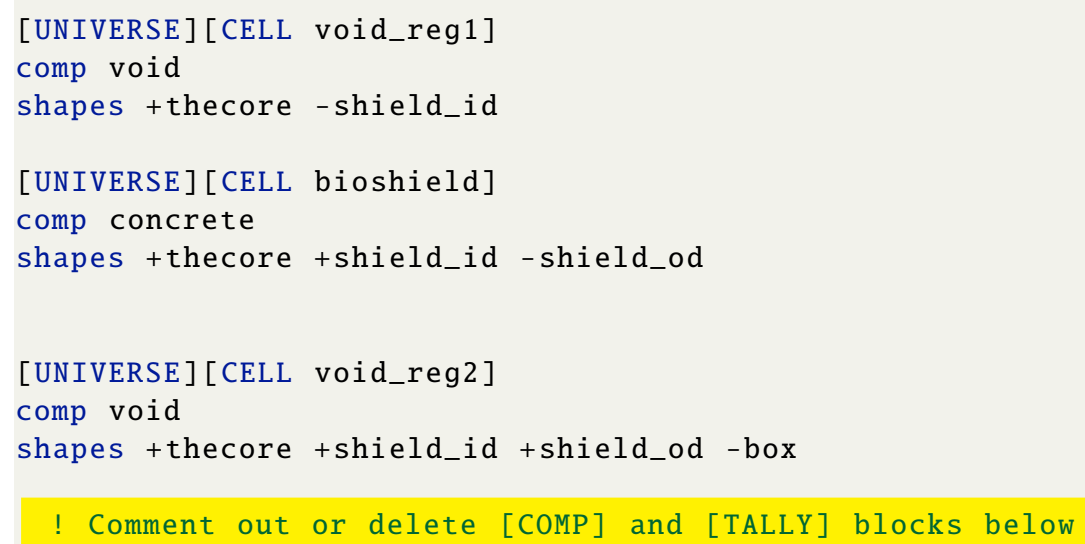

The next step is to run the *. omn excore file using the gg $2 x m l$ executable, /PATH/TO/VERA/INSTALL/bin/gg2xml, located in the bin folder with the VERA build.

$$
\text { /PATH/TO/VERA/INSTALL/bin/gg2xml excore_filename.omn }
$$

This will generate an excore_filename.gg. $x m l$ file. Note that at this step, if there are any input errors that $\mathrm{gg} 2 \mathrm{xml}$ can catch, they will be printed to the terminal. The user can read the errors on the terminal and fix the input as necessary. Instead of typing in the entire path to point to the gg $2 \mathrm{xml}$ executable, the user can create a soft link by typing the following command into the terminal window:

$$
\text { In -s /PATH/TO/VERA/INSTALL/bin/gg2xml }
$$

This will create a soft link to the gg $2 \mathrm{xml}$ executable which can then be invoked by typing the following command into the terminal window:

$$
. / g g 2 \times m l \text { excore_filename.omn }
$$

Once the user has corrected all the input errors and the *.gg.xml file has been successfully generated, the file can be used as an input to the python script, plot.py, which is used to generate the plots to view any further geometry errors (see Listing 12). Note that the user must provide the correct omnibus_gg_xml_filename at the top of the python script. Unfortunately, gg $2 x m l$ will not catch all the input errors in the input parsing step. Once the python script is run by typing the command below into the terminal window, any additional geometry error messages will appear.

python plot.py

In addition to the error messages, a plot called plot 1. png will be output in the working directory. Users can modify this python script as they see fit.

All parts of the input that the user needs to define or for which the user needs to provide input are highlighted in yellow. The lower parameter requires the user to provide the lower bounds of the (x, y, z) axes for plotting purposes, and the upper parameter requires the user to provide the respective upper bounds. This area will be raytraced by Omnibus to check for geometry errors. The sum of all the values in the basis must add up to 1 . If the user would like to generate a plot along a $45^{\circ}$ angle, then the basis should be $(0.707107,0.707107,0)$. 


\section{Listing 12. Python script for geometry error-checking.}

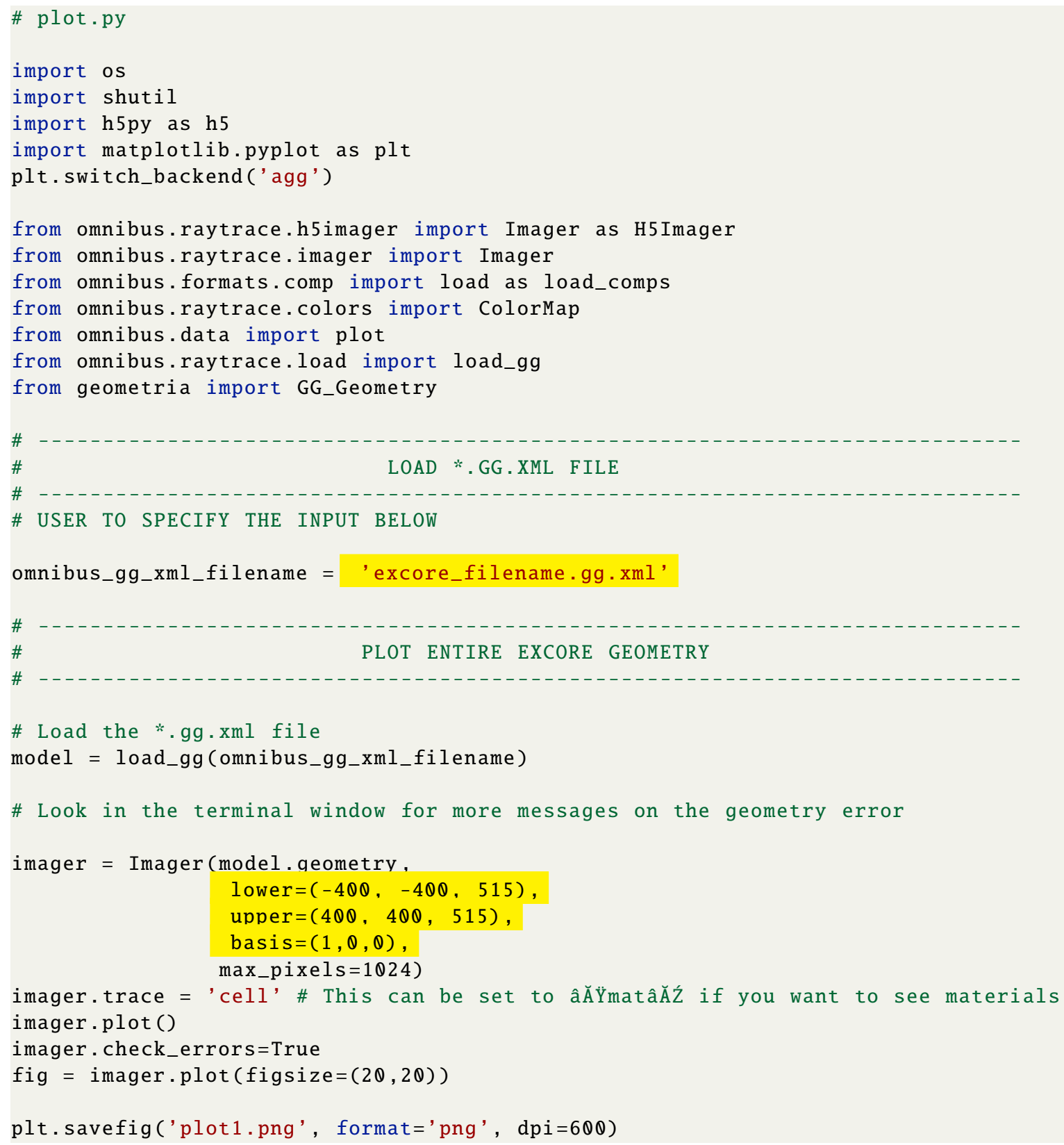

An example of the plot output from plot.py is shown in Figure 9.

Finding all the geometry errors is an iterative process. Once the user is confident that the input is correct, the input that was added to the *. omn file to generate the *.gg. xml file can be commented out or deleted, and the [COMP] and [TALLY] blocks can be added so that it will run through VERA. Note that there may be outstanding geometry errors that will not be caught until the input is run through VERA with the VERA core model integrated. However, most geometry errors should be found at this step with the plot.py script. Note that it is necessary for the user to plot along different basis, upper and lower bounds so that Omnibus will raytrace along multiple planes and directions to find geometry errors. 


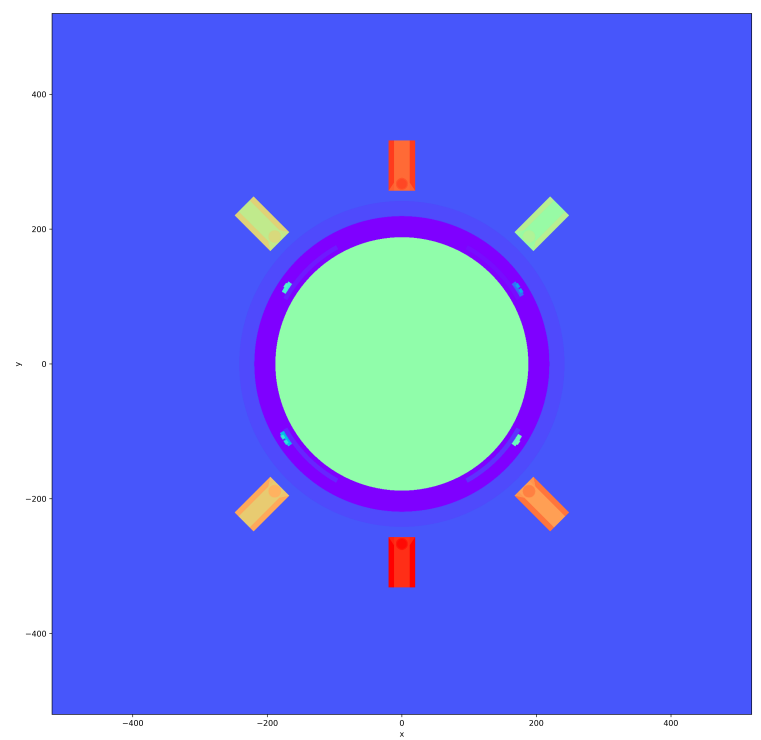

Figure 9. An example of a plot output from plot.py.

\subsection{GENERATING PLOTS AFTER RUNNING VERA}

There are different methods to extract plots and analyze data after running VERA. These tools are discussed further in the following subsections.

\subsubsection{Using VERAView To Examine Pin Adjoints}

In addition to viewing vessel fluence, VERAView can be used to view the pin adjoints. These pin adjoints indicate which pins contribute the most to a vessel or detector response. Figure 10 shows a VERAView screen shot of the pin adjoints for an ex-core detector. The first step is to load the Shift HDF5 output file, vera_output_filename.shift.h5. Note that the user is not opening the VERA output file, vera_output_filename.h5. VERAView will load a 2D core view of the pin adjoints, an axial view as well as the assembly view. The user can click on a specific assembly to view the pin adjoints in greater detail. Shift currently only performs the adjoint calculation at the first statepoint and uses the same adjoint to perform advanced variance reduction throughout the rest of the calculation for subsequent states. The user can slide the Axial bar on the right-hand side to slide between different axial planes to view the adjoint. 


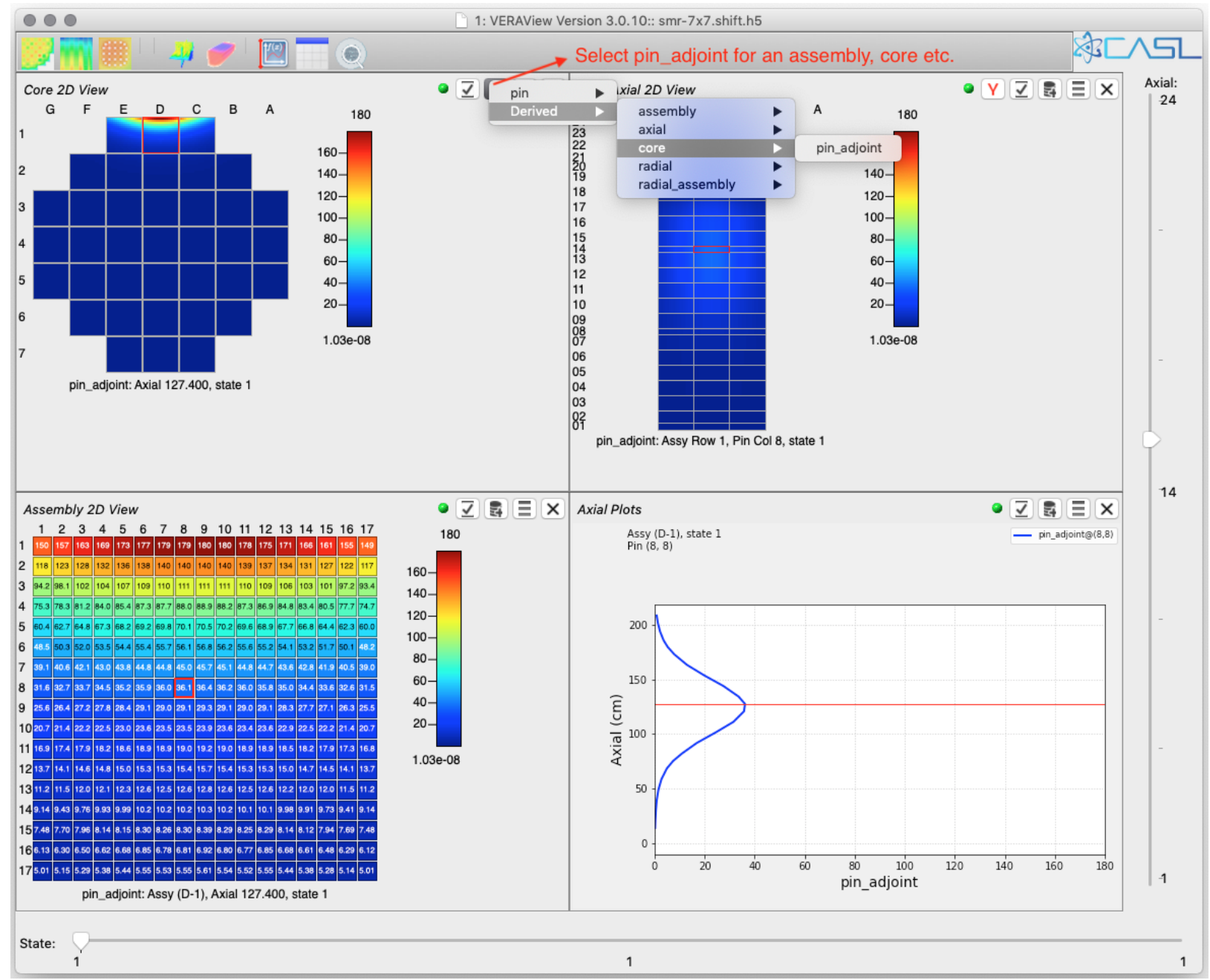

Figure 10. Pin adjoint plots generated with VERAView.

\subsubsection{Custom Python Scripts}

Once VERA finishes running successfully with the ex-core file, the user can use the scripts input.py and plt_adj . py to view the adjoint flux. These scripts provide a basis to the user that can be expanded upon to include additional plotting features.

In the input . py script (see Listing 13), the user must provide the name of the VERA input file without any extensions. Then the user can plot and view the adjoint flux at various locations and provide the zlevel, group number, and the plot file name using the plt_adj.plot_adjoint_flux function. The imager and shift_file should be left as it is in the python script and should not be changed. The zlevel in the example below is set to the same level as the raytrace_levels parameter in the [SHIFT] VERA input. This allows the user to overlay the adjoint flux over the VERA geometry using the python script. Note that the user can overlay the adjoint flux over the geometry of any axial plane (raytrace_levels) but they need to bear in mind that the geometry being overlaid may not capture the features of the axial plane that the adjoint flux represents. To view the total adjoint flux, call the 
plt_adj.plot_tot_adj_flux function in the input.py script, as shown below. The plt_adj .py script contains the functions that process the parameters specified in the input.py file. The user must ensure that both the plt_adj.py and input.py python scripts are in the same working directory as the VERA output. Next, the user can run the set of scripts by typing the following in the terminal:

python input.py

Once again, the user is encouraged to change and customize this script to suit their application. These scripts serve as an example and form a basis for any python scripts the user would like use to produce plots. The plt_adj .py script is not shown here, but it is available with the manual.

\section{Listing 13. Python script for viewing the geometry and adjoint after running VERA.}

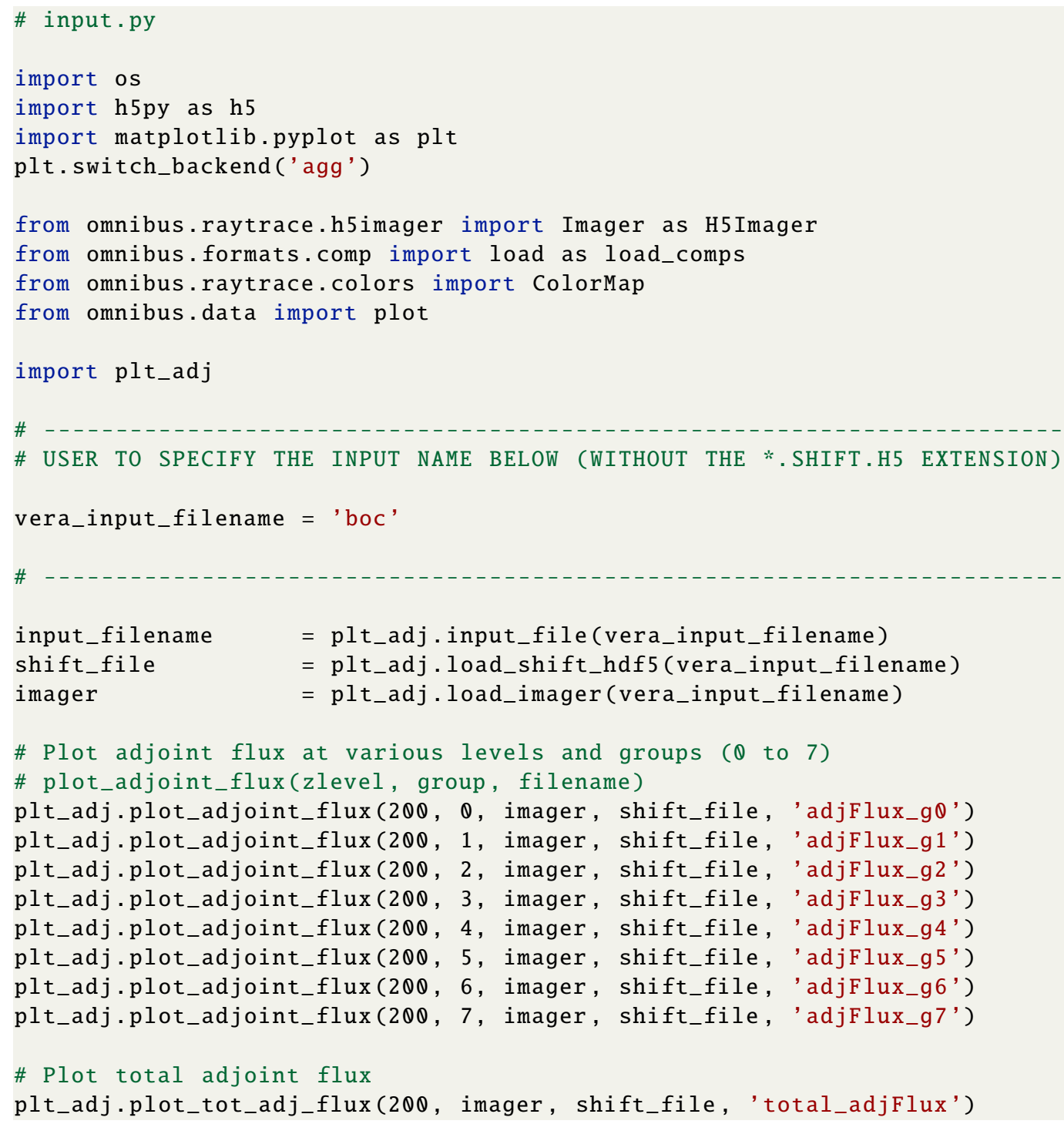

Once the script runs successfully, a folder called Plots is produced. If a folder called Plots already exists, then it must be moved to a different location, renamed, or deleted. In the Plots folder, the user will 
see three items: (1) output_filename.png which shows the integrated in-core and ex-core geometry, (2) a GroupwiseAdjointFlux folder with plots of the groupwise adjoint flux, and (3) a TotalAdjointFlux folder with a plot of the total adjoint flux.

Examples of the plots produced by running input.py are shown in Figure 11.

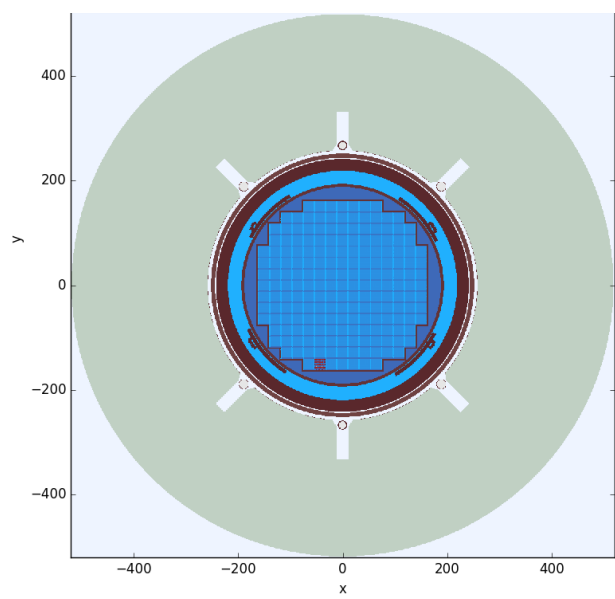

(a) In-core geometry integrated with ex-core geometry.

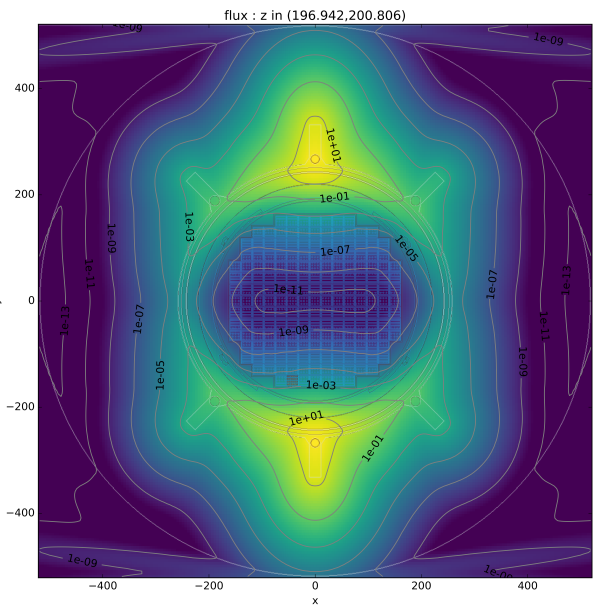

(b) Total adjoint flux.

Figure 11. Integrated in-core and ex-core geometry with the adjoint flux overlaid on the geometry.

\subsection{POST-PROCESSING CELL TALLIES AFTER RUNNING VERA}

A user who is experienced in navigating through HDF5 files can look through the *.h5 VERA output or the *.shift.h5 output to find the ex-core tallies named in the *. omn file. However, this can be cumbersome for users not familiar with HDF5 files, so a python script has been created to facilitate a quick method to pull the ex-core tally data into a text file. This python script is called collate_tally.py, and it requires the user to type the following into the terminal window.

python collate_tally.py vera_output.h5

This will invoke the script to process the HDF5 output files, and it will print out the tally information in a text file called tally.txt (see Listing 14 for an example). The details of the script are not shown in the manual, but they are available for the user with access to VERA.

\section{Listing 14. Python script for viewing the geometry and adjoint after running VERA.}

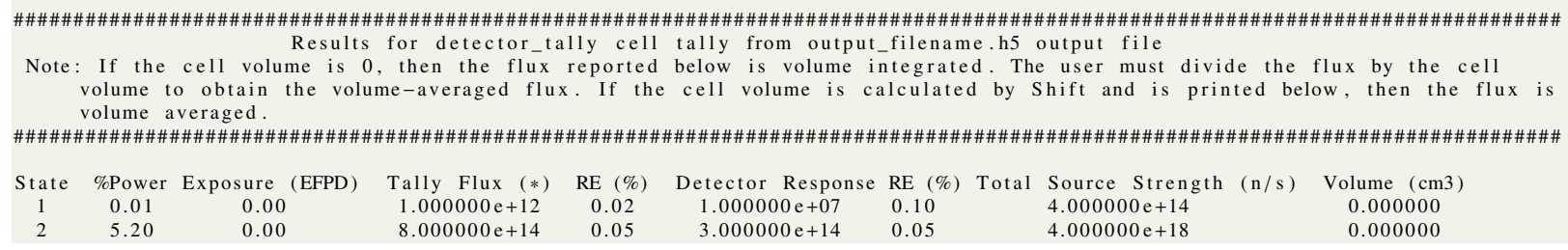

The output prints out the power in percent for each state point in the calculation, the associated exposure in effective full power days (EFPDs), the tally flux and its associated relative error in percent, the detector 
response and its associated percent relative error, the total source strength in units of neutrons per second, and the volume of the tally in cubic centimeters. If the cell volume is zero, then the flux reported under the Tally Flux is volume integrated. Shift is unable to automatically calculate the cell volume because the cell definition was complex, so the user must divide the Tally Flux by the volume of the cell (calculated by the user) to obtain a volume-averaged flux. However, if there is a volume printed out in the output file, then Shift is able to automatically calculate the volume and can divide the cell tally by the volume to provide the user with the volume-averaged tally flux. 



\section{CONCLUSION}

To conclude, this guide serves the purpose of helping the user set up an ex-core calculation using VERA, guiding the user through an ex-core input setup, and providing the user with directions for post-processing results after a calculation. Any feedback from users on this document or the workflow of VERA ex-core calculations is much appreciated. This feedback can be provided through VERA User's Group. The python scripts provided with this user's guide are meant to serve as the basis on which the user can build for their specific applications. 



\section{REFERENCES}

M. N. Avramova. CTF: A Thermal Hydraulic Sub-Channel Code for LWR Transient Analyses, User's

Manual. Technical report, Pennsylvania State University Department of Nuclear Engineering, 2009.

B. Collins, S. Stimpson, B. Kelley, M. Young, B. Kochunas, A. Graham, E. Larsen, T. Downar, and A. Godfrey. Stability and Accuracy of 3D Neutron Transport Simulation Using the 2D/1D Method in MPACT. Journal of Computational Physics, 326:612-628, 2016.

E. E. Davidson, T. M. Pandya, A. T. Godfrey, and M. Asgari. Watts Bar I Ex-Core Analyses Using VERA. In Proceedings of the $20^{\text {th }}$ Topical Meeting of the Radiation Protection Shielding Division, Santa Fe, NM, USA, 2018. American Nuclear Society.

E. E. Davidson, T. M. Pandya, K. E. Royston, T. M. Evans, A. T. Godfrey, Shane C. Henderson, Gary Wolfram, and Joel M. Risner. Effect of Fission Source Spectrum on Monte Carlo Calculation of Ex-core Quantities. In Proceedings of PHYSOR 2020: Transition to a Scalable Nuclear Future, Cambridge, United Kingdom, 2020. American Nuclear Society.

S. Johnson, T. Evans, G. Davidson, S. Hamilton, T. Pandya, K. Royston, and E. Biondo. Omnibus User Manual. Technical Report ORNL/TM-2018/1073, ORNL, TBD 2020.

S. Palmtag and A. T. Godfrey. VERA Common Input User Manual. Technical Report CASL-U-2014-0014-002, CASL, February 2015.

T. Pandya, T. Evans, K. Royston, K. Clarno, B. Collins, S. Stimpson, and S. Henderson. VERAShift User's Manual. Technical Report CASL-U-2019-1921-000, CASL, January 2020.

T. M. Pandya, S. R. Johnson, T. M. Evans, G. G. Davidson, S. P. Hamilton, and A. T. Godfrey. Implementation, Capabilities, and Benchmarking of Shift, a Massively Parallel Monte Carlo Radiation Transport Code. Journal of Computational Physics, 308:239-272, 2016.

H. P. Smith, E. E. Davidson, A. T. Godfrey, and T. M. Pandya. An Analysis of Various Solution Strategies and Perturbations on Inputs of the Reactor Shielding Problem. In Proceedings of the $20^{\text {th }}$ Topical Meeting of the Radiation Protection Shielding Division, Santa Fe, NM, USA, 2018. American Nuclear Society.

J. A. Turner, K. Clarno, M. Sieger, R. Bartlett, B. Collins, R. Pawlowski, R. Schmidt, and R. Summers. The Virtual Environment for Reactor Applications (VERA): Design and Architecture. Journal of Computational Physics, 326:544-568, December 2016.

J. C. Wagner and A. Haghighat. Automated Variance Reduction of Monte Carlo Shielding Calculations Using the Discrete Ordinates Adjoint Function. Nuclear Science and Engineering, 128(2):186-208, 1998. 